TAIWANESE JOURNAL OF MATHEMATICS

Vol. 4, No. 2, pp. 215-252, June 2000

\title{
LIE ISOMORPHISMS IN *-PRIME GPI RINGS WITH INVOLUTION
}

\author{
Philip S. Blau and Wallace S. Martindale, 3rd
}

\begin{abstract}
Let $R$ and $S$ be *-prime GPI rings with involution, with respective skew elements $K$ and $L$, with respective extended centroids $C$ and $D$, and let $\alpha:[K, K] /[K, K] \cap C \rightarrow[L, L] \cap D$ be a Lie isomorphism. If both involutions are of the second kind it is shown that $\alpha$ is determined by a related associative isomorphism and if the involutions are of different kinds it is shown that such a map $\alpha$ cannot exist (modulo some lowdimensional counterexamples).
\end{abstract}

\section{Statements of the Main Results}

Let $R$ be an associative ring with involution *, i.e., an antiautomorphism of period 1 or 2. An ideal $I$ of $R$ is a ${ }^{*}$-ideal if $I^{*}=I$, and $R$ is said to be ${ }^{*}$-prime if the product of any two nonzero *-ideals of $R$ is again nonzero. Let $K=\left\{x \in R \mid x^{*}=-x\right\}$ denote the Lie ring of skew elements of $R$, and let $C$ be the extended centroid of $R$. An involution is induced on $C$ as follows: for $c \in C, c^{*} u=\left(c u^{*}\right)^{*}, u \in U, U$ a nonzero *-ideal of $R$ such that $c U \subseteq R$. The involution * on $R$ is said to be of the first kind if the involution induced on $C$ is the identity map; otherwise ${ }^{*}$ is of the second kind. Accordingly, as in $[10$, p.27], we shall say that $R$ is of type 1 (resp. type 2) if the involution on $R$ is of the first kind (resp. second kind).

Clearly, a *-prime ring is either already a prime ring or contains a *-ideal of the form $T \oplus T^{*}, T$ an ideal of $R$ which is itself a prime ring. We shall say that a *-prime ring is GPI (= generalized polynomial identity) if $R$ is GPI in the usual sense in case $R$ is prime or the component $T$ (above) is GPI in case $R$ is not prime.

Received November 6, 1998; revised June 3, 1999.

Communicated by P.-H. Lee.

2000 Mathematics Subject Classification: 16W10, 16W20.

Key words and phrases:*-prime GPI ring, Lie isomorphism. 
Throughout this paper we make the blanket assumption that all rings encountered are 2-torsion free, and we will not repeat this remark. We also mention here that for any subset $T$ of a ring the notation $\langle T\rangle$ will indicate the associative subring generated by $T$.

In this paper we are interested in characterizing Lie isomorphisms of Lie rings of the form $\overline{[K, K]}=[K, K] /[K, K] \cap C, K$ the skew elements of a *-prime GPI ring.

In case $R$ is a simple ring with involution, the earlier Herstein theory (see, e.g., $[4$, Chapter 2]) shows that $\overline{[K, K]}$ is a simple Lie ring (modulo some low dimensional cases). In the wider context of *-prime rings it has been shown (see, e.g., $[3,4,5]$ ) that $\overline{[K, K]}$ is a prime Lie ring in the sense that the Lie product of any two nonzero Lie ideals of $\overline{[K, K]}$ remains nonzero. Thus $\overline{[K, K]}$ appears to be a natural Lie ring to study.

To make matters more specific, let $R$ and $S$ be *-prime rings with respective extended centroids $C$ and $D$, and with respective skew elements $K$ and $L$. We consider Lie isomorphisms

$$
\alpha: \overline{[K, K]} \rightarrow \overline{[L, L]}
$$

If $R$ and $S$ are of the same type, the natural conjecture is as follows: there is a ring monomorphism $\psi:\langle[K, K]\rangle \longrightarrow S D+D$ such that for $x \in[K, K]$

$$
x^{\psi}=y+\rho, y \in[L, L], \rho \in D, \bar{y}=\bar{x}^{\alpha} .
$$

We note that in (2) $y$ and $\rho$ are unique up to elements of $[L, L] \cap D$ and in this sense one may say that $\alpha$ is determined by $\psi$. We also remark that unless $R$ satisfies $S_{4},\langle[K, K]\rangle$ contains a nonzero ${ }^{*}$-ideal of $R$, so in this sense $\psi$ acts on a "large" piece of $R$.

For $R$ and $S$ both GPI of type 1, the conjecture (modulo some low dimension exceptions) has been earlier verified [9, Corollary 3.2]. For $R$ and $S$ not GPI, each of type 2, and one of them containing three orthogonal symmetric idempotents adding up to 1 , the conjecture has been verified by M. Rosen [11, Theorem 5.1]. Therein lies part of the motivation for this paper: to complement Rosen's result when at least one of $R$ and $S$ is GPI and both are of type 2. As one of the main results of this paper we now state the following.

Theorem 1.1. Let $R$ and $S$ be ${ }^{*}$-prime rings of type 2, with respective skew elements $K$ and $L$ and with respective extended centroids $C$ and $D$. We assume:

1. At least one of $R$ and $S$ is GPI.

2. At least one of $R$ and $S$ does not satisfy $S_{4}($ if char. $\neq 3)$ or $S_{6}$ (if char. $=3)$. 
Let $\overline{[K, K]}=[K, K] /[K, K] \cap C$ and $\overline{[L, L]}=[L, L] /[L, L] \cap D$. Suppose there is a Lie isomorphism

$$
\alpha: \overline{[K, K]} \rightarrow \overline{[L, L]} .
$$

Then there exists a ring monomorphism

$$
\psi:\langle[K, K]\rangle \rightarrow S D+D
$$

such that for all $x \in[K, K]$,

$$
x^{\psi}=y+\rho, y \in[L, L], \rho \in D \cap L D_{*}, \bar{y}=\bar{x}^{\alpha} .
$$

Furthermore, letting $T^{(2)}$ denote $[[T, T],[T, T]]$, we have

(i) $\left(K^{(2)}\right)^{\psi}=L^{(2)}\left(\right.$ whence $\left.\left\langle K^{(2)}\right\rangle^{\psi}=\left\langle L^{(2)}\right\rangle\right)$.

(ii) For any nonzero ${ }^{*}$-ideal $I$ of $R$ in $\left\langle K^{(2)}\right\rangle$ (such exist), $I^{\psi}$ contains a nonzero *-ideal of $S$.

We remark that the question of settling the conjecture when neither $R$ nor $S$ is GPI is being addressed in [2] (currently in preparation at this writing).

The other motivation for this paper is to investigate what happens when $R$ and $S$ are of different types, a situation we shall sometimes refer to as the "mixed" case. Roughly speaking, the result we obtain is: if $R$ and $S$ are GPI but of different types, then, with two "low dimensional" exceptions, there does not exist a Lie isomorphism $\alpha: \overline{[K, K]} \rightarrow \overline{[L, L]}$. An accurate statement of this result will be given later in this section, after we proceed to review further notions and basic results pertaining to ${ }^{*}$-prime rings.

Let $R$ be a ${ }^{*}$-prime ring with extended centroid $C . C_{*}=\left\{c \in C \mid c^{*}=c\right\}$ is called the ${ }^{*}$-extended centroid of $R$ and is easily seen to be a field. Clearly, $R$ is of type 1 (resp. type 2) if and only if $C=C_{*}$ (resp. $C=C_{*}+\beta C_{*}, \beta^{*}=$ $-\beta \neq 0)$. A *-prime ring $R$ is ${ }^{*}$-closed if it is already an algebra over $C_{*}$. For example $R C_{*}$ is a ${ }^{*}$-closed ${ }^{*}$-prime algebra over $C_{*}$ and accordingly is called the ${ }^{*}$-closure of $R$. We cite the following useful result which is instrumental in transferring some problems about *-closed *-prime rings to closed prime rings. Part (a) is a restatement of [10, Theorem 2.11(a)] and part (b) is a straightforward exercise using part (a) in conjunction with well-known facts about the socle.

Remark 1.2. Let $R$ be a ${ }^{*}$-closed ${ }^{*}$-prime algebra over $C_{*}$ which is not prime. Then:

(a) There exists a ${ }^{*}$-ideal $J \oplus J^{*}$, where $J$ is a closed prime algebra over $C_{*}$.

(b) If $R$ is GPI there exists a unique *-ideal $H \oplus H^{*}$, where $H$ is its own socle and is simple with centroid $C_{*}\left(H \oplus H^{*}\right.$, which is *-simple, will be called the ${ }^{*}$-socle of $R$ ). 
In the course of this paper various problems will be reduced to the case of matrix rings $M_{n}(E)$ over a field $E$. For instance, when studying the mixed case, it is especially useful to let $E$ be an algebraically closed field, since in this case it is well-known that any involution of the first kind on $M_{n}(E)$ is either transpose or symplectic. A first step in the passage toward bringing $M_{n}(E)$ into play is to form what is called [1, p. 945] the super *-closure of a *-prime ring $R$, namely,

$$
A=R C_{*} \otimes_{C_{*}} E,
$$

where $E$ is the algebraic closure of $C_{*}$. The involution ${ }^{*}$ on $R$ can be lifted uniquely to an involution ${ }^{*}$ on $A$ via the rule

$$
\sum x_{i} c_{i} \otimes \lambda_{i} \rightarrow \sum x_{i}^{*} c_{i} \otimes \lambda_{i}
$$

where $x_{i} \in R, c_{i} \in C_{*}, \lambda_{i} \in E$. Making tacit use of Remark 1.2 combined with standard ring theory techniques, we proceed to state some useful facts concerning $A$. First, the extended centroid $C(A)=C \otimes_{C_{*}} E$. If $\beta$ is a nonzero skew element of $C$, then $e=1 / 2\left(1 \otimes 1+\beta \otimes \beta^{-1}\right)$ is an idempotent in $C(A)$ such that $e \oplus e^{*}=1$, where $e^{*}=1 / 2\left(1 \oplus 1-\beta \otimes \beta^{-1}\right)$. $A$ is ${ }^{*}$-closed ${ }^{*}$-prime over $E \cong C_{*} \otimes_{C_{*}} E$ with skew elements $M=K C_{*} \otimes E$. If $R$ is of type 2 , then $A$ contains a nonzero *-ideal of the form $J \oplus J^{*}$, where $\mathrm{J}$ is a closed prime algebra over $E \cong e E$. In case $R$ is GPI of type $2, J \oplus J^{*}$ may be taken to be the ${ }^{*}$-socle $G \oplus G^{*}$, where $G$ is a centroid simple algebra over $E \cong e E$ which is a dense ring of finite-rank linear transformations of a vector space over $E$.

We now state our second main result in more detail.

Theorem 1.3. Let $R$ be $a{ }^{*}$-prime ring of type 1 , with skew elements $K$ such that $[K, K] \neq 0$, and with super ${ }^{*}$-closure $A=R C \otimes_{C} E$. Let $S$ be a ${ }^{*}$-prime ring of type 2 , with skew elements $L$ such that $[L, L] \neq 0$, and with super ${ }^{*}$-closure $B=S D_{*} \otimes_{D_{*}} F$. Assume that at least one of $R$ and $S$ is GPI. Then there does not exist a Lie isomorphism $\alpha:[K, K] \rightarrow \overline{[L, L]}$ except in the following two situations:

(i) $A=M_{3}(E)$ with * transpose; $A=M_{2}(E)$ with * symplectic; $B=$ $H \oplus H^{*}, H=M_{2}(F)$.

(ii) $A=M_{6}(E)$ with ${ }^{*}$ transpose; $B=H \oplus H^{*}, H=M_{4}(F)$.

Lie isomorphisms illustrating (i) and (ii) in Theorem 1.3 do in fact occur (see [7, p. 142]).

The remaining sections in this paper are all motivated toward proving Theorems 1.1 and 1.3. In Section 2, making strong use of the intimate connection between $C_{*}$ and the extended centroid of the prime Lie ring $\overline{[K, K]}$ proved in [1], we show (Lemma 2.4) that $\alpha$ may be lifted to a Lie isomorphism of 
the derived skew elements (modulo the respective centers) of the respective super *-closures. In Section 3, a pot pourri of useful results is established. This includes how Lie isomorphisms act on square-zero elements (Lemmas 3.4 and 3.5) and also contains a statement of Howland's Theorem (Theorem 3.7), which is the noninvolution analogue of Theorem 1.1. The purpose of Section 4 is to show that if one of the two rings is GPI then both are GPI (Theorem 4.1), thereby projecting a useful symmetry into the situation. In Sections 5 and 6 , the proof of Theorem 1.1 is given provided the rings are their own super *-closures: in Section 5 when the rings are GPI but not PI (Theorem 5.2) and in Section 6 when the rings are PI (Theorem 6.2). The proof of Theorem 1.1 is then completed in Section 7. Section 8 provides useful structural results (Lemmas 8.1 and 8.2) which are then used in Section 9 to prove Theorem 1.3.

\section{Extending Lie Isomorphisms}

Let $R$ be a *-prime ring with skew elements $K$, extended centroid $C$, *-extended centroid $C_{*}$, and super *-closure $A=R C_{*} \otimes E$, where $E$ is an algebraic closure of $C_{*}$. We have noted in Section 1 that $A$ is a ${ }^{*}$-closed *-prime algebra over $E$ with an involution extending *:

$$
x c \otimes \lambda \rightarrow x^{*} c \otimes \lambda, x \in R, c \in C_{*}, \lambda \in E .
$$

We assume that $[K, K] \neq 0$ and that $A \neq M_{4}(E)$ under transpose, since with these restrictions in place it is implicit from [1, Theorem 5.2] that $\overline{[K, K]}=$ $[K, K] /[K, K] \cap C$ is a prime Lie ring. In a similar manner, we let $S$ be a *-prime ring with skew elements $L$, extended centroid $D,{ }^{*}$-extended centroid $D_{*}$, and super *-closure $B=S D_{*} \otimes F$, where $F$ is a (suitably chosen) algebraic closure of $D_{*}$. Again we assume $[L, L] \neq 0$ and $B \neq M_{4}(F)$ under transpose. We now suppose that we are given a Lie isomorphism

$$
\alpha: \overline{[K, K]} \rightarrow \overline{[L, L]}
$$

Our first goal is to show that $\alpha$ may be extended to a Lie isomorphism of $\overline{[K, K] C_{*}}$ onto $\overline{[L, L] D_{*}}$. The initial step in this regard is to show that $C_{*} \cong D_{*}$ in a way compatible with $\alpha$. The idea is to make use of the fact $[1$, Theorem $5.2]$ that $C_{*} \cong C(\overline{[K, K]})$ (the extended centroid of the prime Lie ring $\overline{[K, K]}$ ) and that $C(\overline{[K, K]}) \cong C(\overline{[L, L]})$ in a natural way using $\alpha$. This is made precise in

Lemma 2.1. Given a Lie isomorphism $\alpha: \overline{[K, K]} \rightarrow \overline{[L, L]}$, there exists an isomorphism $\gamma: C_{*} \rightarrow D_{*}$ which is compatible with $\alpha$ in the following sense: 
For $c \in C_{*}$, there exists a Lie ideal $U$ of $K, U \subseteq[K, K], U \nsubseteq C$, and a Lie ideal $V$ of $L, V \subseteq[L, L]$, such that

$$
\bar{V}=\bar{U}^{\alpha}, \quad c U \subseteq[K, K]
$$

and for all $u \in U$

$$
\overline{c^{\gamma} v}=\bar{w}, \quad v \in V,
$$

where $w \in[L, L], \bar{w}=\overline{c u}^{\alpha}, \bar{v}=\bar{u}^{\alpha}$.

Sketch of proof. We define $\gamma$ as the composite of the following three isomorphisms:

$$
C_{*} \stackrel{\phi}{\rightarrow} C(\overline{[K, K]}) \stackrel{\sigma}{\rightarrow} C(\overline{[L, L]}) \stackrel{\rho}{\rightarrow} D_{*} .
$$

We shall indicate how $\phi, \sigma$, and $\rho$ are defined but will leave the details of proof to the reader, whom we refer to [1, Theorem 5.2], where it is to be noted that $\phi$ is in essence the composite $\phi_{1} \phi_{2} \phi_{3}$ and $\rho$ is just $\phi_{4}$.

Writing $c=[g: I \rightarrow R] \in C_{*}, I$ a ${ }^{*}$-ideal, one defines $c^{\phi}=[h: \bar{U} \rightarrow$ $\overline{[K, K]}] \in C(\overline{[K, K]})$, where $U=[I \cap K, K]$ and $h(\bar{u})=\overline{c u}, u \in U$.

For $c=[h: \bar{U} \rightarrow \overline{[K, K]}] \in C(\overline{[K, K]})$ one defines $c^{\sigma}=[p: \bar{V} \rightarrow \overline{[L, L]}] \in$ $C(\overline{[L, L]})$, where $V$ is a Lie ideal of $[L, L]$ such that $\bar{V}=\bar{U}^{\alpha}$ and $p\left(\bar{u}^{\alpha}\right)=h(\bar{u})^{\alpha}$.

For $c=[p: \bar{V} \rightarrow \overline{[L, L]}] \in C(\overline{[L, L]})$, one defines $c^{\rho}=\left[f: S^{\prime} T S^{\prime} \rightarrow\right.$ $S] \in C(\overline{[L, L]})$, where $T=[V,[L, L]]$ and $f$ is given according to $f([v, y])=$ $[w, y], w \in[L, L], \bar{w}=p(\bar{v})$. The main problem involved with $\rho$ is showing that it is well-defined; in fact, most of the paper [1] is devoted to this purpose.

A careful examination of the composite of these three isomorphisms results in the statement of the lemma.

In case $[K, K]$ and $[L, L]$ have no nonzero central elements, the statement of Lemma 2.1 becomes greatly simplified:

Corollary 2.2. If $[K, K] \cap C=0$ and $[L, L] \cap D=0$ and $\alpha:[K, K] \rightarrow$ $[L, L]$ is a Lie isomorphism, then there is an isomorphism $\gamma: C_{*} \rightarrow D_{*}$ such that for all $c \in C_{*}$ there exists a nonzero Lie ideal $U$ of $K, U \subseteq[K, K]$, such that for all $u \in U, c^{\gamma} u^{\alpha}=(c u)^{\alpha}$.

Lemma 2.3. Any Lie isomorphism $\alpha: \overline{[K, K]} \rightarrow \overline{[L, L]}$ may be extended to a $\gamma$-semilinear Lie isomorphism (again denoted by) $\alpha: \overline{[K, K] C_{*}} \rightarrow \overline{[L, L] D_{*}}$ given by

$$
\overline{\sum x_{i} c_{i}} \rightarrow \overline{\sum y_{i} c_{i}^{\gamma}}
$$

where $x_{i} \in[K, K], c_{i} \in C_{*}, y_{i} \in[L, L], \bar{y}_{i}={\overline{x_{i}}}^{\alpha}$. 
(Note: It is to be understood that the bars in (3) refer to the moduli $[K, K] C_{*} \cap$ $C$ and $[L, L] D_{*} \cap D$.)

Proof. We show that this extension is well-defined, leaving the remaining straightforward details to the reader. To this end, suppose $\overline{\sum x_{i} c_{i}}=\overline{0}$, i.e., $\sum x_{i} c_{i}$ is central. By Lemma 2.1 (applied simultaneously to the finite number of $c_{i}$ 's), there exists a Lie ideal $U$ of $K, U \subseteq[K, K], U \nsubseteq C$, and a Lie ideal $V$ of $L, V \subseteq[L, L]$, such that $\bar{V}=\bar{U}^{\alpha}, c_{i} U \subseteq[K, K]$, where for all $u \in U c_{i}^{\gamma} v=$ $w_{i}, w_{i} \in[L, L], \overline{w_{i}}={\overline{c_{i} u}}^{\alpha}$. For all $u \in U, 0=\left[\sum x_{i} c_{i}, u\right]=\sum\left[x_{i}, c_{i} u\right]$ and so $\sum\left[\overline{x_{i}}, \overline{c_{i} u}\right]=\overline{0}$. Applying $\alpha$ we have

$$
\overline{0}=\sum\left[{\overline{x_{i}}}^{\alpha},\left(\overline{c_{i} u}\right)^{\alpha}\right]=\sum\left[\overline{y_{i}}, \overline{c_{i}^{\gamma} v}\right]=\overline{\sum\left[y_{i}, c_{i}^{\gamma} v\right]} .
$$

Therefore, $\sum\left[y_{i}, c_{i}^{\gamma} v\right] \in[L, L] \cap D$ whence $\left[\sum y_{i} c_{i}^{\gamma}, V D_{*}\right] \subseteq[L, L] D_{*} \cap D$. This says that $\sum y_{i} c_{i}^{\gamma} \in D$ (by the primeness of $[L, L] D_{*}$ ) and so $\overline{\sum y_{i} c_{i}^{\gamma}}=\overline{0}$.

Finally, it will be useful (especially in Sections 4 and 9) to extend $\alpha$ to a Lie isomorphism of the derived skew elements (modulo the center) of the super *-closures of $R$ and $S$. We let $M=K C_{*} \otimes E$ and $N=L D_{*} \otimes F$ denote the respective skew elements of the super ${ }^{*}$-closures $A$ and $B$.

Lemma 2.4. Any Lie isomorphism $\alpha: \overline{[K, K]} \rightarrow \overline{[L, L]}$ may be extended to a $\gamma$-semilinear Lie isomorphism (again denoted by) $\alpha: \overline{[M, M]} \rightarrow \overline{[N, N]}$ given by

$$
\overline{\sum x_{i} c_{i} \otimes \lambda_{i}} \rightarrow \overline{\sum y_{i} c_{i}^{\gamma} \otimes \lambda_{i}^{\gamma}}
$$

where $x_{i} \in[K, K], c_{i} \in C_{*}, y_{i} \in[L, L], \overline{y_{i}}={\overline{x_{i}}}^{\alpha}$ and $\gamma: E \rightarrow F$ is an extension of $\gamma: C_{*} \rightarrow D_{*}$.

(Note: The bars in (4) refer to the moduli $[M, M] \cap(C \otimes E)$ and $[N, N] \cap(D \otimes$ $F)$.)

Proof. $\overline{[M, M]}$ and $\overline{[N, N]}$ may be identified respectively with $\overline{[K, K] C_{*}} \otimes E$ and $\overline{[L, L] D_{*}} \otimes F$. By Lemma 2.3, $\alpha$ may first be extended to a $\gamma$-semilinear Lie isomorphism $\alpha: \overline{[K, K] C_{*}} \rightarrow \overline{[L, L] D_{*}}$ according to $\overline{x c} \rightarrow \overline{y c^{\gamma}}, x \in$ $[K, K], c \in C_{*}, y \in[L, L], \bar{y}=\bar{x}^{\alpha}$. By usual tensor product considerations, $\alpha$ may then be further extended to the required map $\alpha \otimes \gamma$ (which we again just denote as $\alpha$ ). 


\section{Useful Background Results}

In this section, we present a variety of results which play a useful role in the arguments appearing in the subsequent sections.

We begin with two (undoubtedly well-known) results concerning rings which are their own socle. For the first two lemmas, $H$ is assumed to be a dense ring of finite-rank linear transformations of a vector space $V$ over a field $F$, i.e., $H$ is a simple ring which is its own socle and the commuting ring is the field $F$.

Lemma 3.1. If $a \in H$ but $a \notin[H, H]$, then $H=[H, H]+F a$.

Proof. If $(V: F)$ is finite, we have $H=M_{n}(F)$, in which case the result is obvious since $\operatorname{dim}[H, H]=n^{2}-1$. Thus we may suppose that $(V: F)=\infty$. Now let $a \notin[H, H]$ be given and let $x$ be an arbitrary element of $H$. By Litoff's Theorem [3, Theorem 4.3.11], there exists an idempotent $e$ in $H$ such that both $a$ and $x$ belong to $e H e \cong M_{k}(F)$. By the finite-dimensional case above, we may write $e H e=[e H e, e H e]+F a$. Thus $x=e x e \in[H, H]+F a$ and we are done.

Lemma 3.2. Let $a \in[H, H]$ but not central. Then there exists $x \in[H, H]$ such that $x a \notin[H, H]$.

Proof. We first assume that $(V: F)<\infty$, i.e., $H=M_{n}(F)$. We write $a=b+c$, where $b=\sum_{i=1}^{n} \alpha_{i i} e_{i i}$ and $c=\sum_{i \neq j} \alpha_{i j} e_{i j}, \alpha_{i j} \in F,\left\{e_{i j}\right\}$ the usual matrix units. Suppose $c \neq 0$, say, $\alpha_{12} \neq 0$. Setting $x=e_{21}$, we have $e_{21} a=\alpha_{11} e_{21}+\alpha_{12} e_{22}+\sum_{j=3}^{n} \alpha_{1 j} e_{2 j}$. Therefore the trace of $e_{21} a=\alpha_{12} \neq 0$ and so $e_{21} a \notin[H, H]$. If $c=0$, we may assume without loss of generality that $\alpha_{11} \neq \alpha_{22}$ (otherwise $a$ would be central). Setting $x=e_{11}-e_{22}=\left[e_{12}, e_{21}\right] \in$ $[H, H]$, we see that $x a=\alpha_{11} e_{11}-\alpha_{22} e_{22}$. Thus the trace of $x a=\alpha_{11}-\alpha_{22} \neq 0$, whence $x a \notin[H, H]$.

We next assume that $(V: F)=\infty$. By Litoff's Theorem, there exists an idempotent $e \in H$ such that $a \in e H e$ but $a$ does not lie in the center of $e H e$. By the finite-dimensional case just proved, we may pick $x \in[e H e, e H e] \subseteq[H, H]$ such that $x a \notin[e H e, e H e]$. Suppose, however, that $x a \in[H, H]$. Then $x a=\sum_{i=1}^{m}\left[h_{i}, g_{i}\right], h_{i}, g_{i} \in H$. By Litoff's Theorem again, there is an idempotent $f \in H$ such that $e, h_{1}, g_{1}, \ldots, h_{m}, g_{m} \in f H f$. Thus $x a \in[f H f, f H f]$. Therefore the trace of $x a$ in $f H f$ is equal to the trace of $x a$ in $e H e$ since $x a \in e H e$. This contradicts $x a \notin[e H e, e H e]$, and the lemma is proved. 
We turn our attention next to a useful general result on tensor products of vector spaces.

Lemma 3.3. Let $V$ be a vector space over a field $F$, and let $r_{1}, r_{2}, \ldots, r_{m}, s_{1}, s_{2}, \ldots$, $s_{m} \in V$ with $r_{1}, r_{2}, \ldots, r_{m}$ independent. Suppose, for some $\lambda_{1}, \ldots, \lambda_{m} \in F$,

$$
\sum_{i=1}^{m} r_{i} \otimes s_{i}+\sum_{i=1}^{m} \lambda_{i} s_{i} \otimes r_{i}=0
$$

Then, for all $i=1,2, \ldots, m, s_{i} \in s p\left\{r_{1}, r_{2}, \ldots, r_{m}\right\}$.

Proof. By a suitable reordering, let $r_{1}, r_{2}, \ldots, r_{m}, s_{1}, \ldots, s_{k}$ be a maximal independent subset of $r_{1}, \ldots, r_{m}, s_{1}, \ldots, s_{m}$. We may assume that $k<m$ (otherwise this would force $\left.s_{1}=s_{2}=\ldots=s_{m}=0\right)$. For $l=k+1, \ldots, m$, we write

$$
s_{l}=\sum_{i=1}^{m} \alpha_{l i} r_{i}+\sum_{j=1}^{k} \beta_{l j} s_{j} .
$$

Substituting (6) in (5), we have

$$
\begin{aligned}
& \sum_{i=1}^{m} r_{i} \otimes s_{i}+\sum_{j=1}^{k} s_{j} \otimes \lambda_{j} r_{j}+\sum_{l=k+1}^{m}\left(\sum \alpha_{l i} r_{i} \otimes \lambda_{l} r_{l}\right)+\sum_{l=k+1}^{m}\left(\sum_{j=1}^{k} \beta_{l j} s_{j} \otimes \lambda_{l} r_{l}\right) \\
& =\sum_{i=1}^{m} r_{i} \otimes\left[s_{i}+\sum_{l=k+1}^{m} \alpha_{l i} \lambda_{l} r_{l}\right]+\sum_{j=1}^{k} s_{j} \otimes\left[\lambda_{j} r_{j}+\sum_{l=k+1}^{m} \beta_{l j} \lambda_{l} r_{l}\right]=0 .
\end{aligned}
$$

In particular, this forces $s_{i}=-\sum_{l=k+1}^{m} \alpha_{l i} \lambda_{l} r_{l}, i=1,2, \ldots, m$, and the proof is complete.

In several places in this paper it is important to know that (roughly speaking) square-zero elements are mapped to square-zero elements under a Lie isomorphism. In Lemmas 3.4 and 3.5, Lie compatible conditions are given which assure that an element essentially has square zero. In Lemma 3.6, conditions are given which assure that the product of two square-zero elements is essentially zero.

For the next three lemmas, $B$ will denote a closed prime algebra over a field $F$. For $a \in B$, we denote by $a_{r}$ and $a_{l}$ the respective right and left multiplications of $B$ determined by $a$.

Lemma 3.4. Suppose char. $F \neq 3$ and let a be a noncentral element of $B$ such that $\left(a_{r}-a_{l}\right)^{4}=0$. Then there exists a unique $\lambda \in F$ for which $(a-\lambda)^{2}=0$. 
Proof. Using the well-known isomorphism $B_{l} B_{r} \cong B^{o} \otimes_{F} B$, the condition $\left(a_{r}-a_{l}\right)^{4}=0$ translates to

$$
1 \otimes a^{4}-4 a \otimes a^{3}+6 a^{2} \otimes a^{2}-4 a^{3} \otimes a+a^{4} \otimes 1=0 .
$$

By Lemma 3.3, we see in particular that

$$
a^{3}=\alpha+\beta a+\gamma a^{2}, \alpha, \beta, \gamma \in F,
$$

and hence that

$$
a^{4}=\gamma \alpha+(\alpha+\gamma \beta) a+\left(\beta+\gamma^{2}\right) a^{2} .
$$

Suppose 1,a, $a^{2}$ are F-independent. Substitution of (9) in (7) yields

$$
\begin{aligned}
1 \otimes\left[\gamma \alpha+(\alpha+\gamma \beta) a+\left(\beta+\gamma^{2}\right) a^{2}\right] & \\
-4 a \otimes\left[\alpha+\beta a+\gamma a^{2}\right] & \\
+6 a^{2} \otimes a^{2}-4 a^{3} \otimes a+a^{4} \otimes 1 & =0 .
\end{aligned}
$$

Rearrangement of the terms in (10) leads to

$$
[\quad] \otimes 1+[\quad] \otimes a+\left[\left(\beta+\gamma^{2}\right)-4 \gamma a+6 a^{2}\right] \otimes a^{2}=0 .
$$

This forces $6 a^{2}=4 \gamma a-\left(\beta+\gamma^{2}\right)$, which, because char. $F \neq 3$, contradicts $1, a, a^{2}$ being independent. Therefore $1, a, a^{2}$ are dependent, and since $1, a$ are independent, we may write

$$
a^{2}=\lambda+\mu a, \quad \lambda, \mu \in F .
$$

From (11), we obtain

$$
\begin{gathered}
a^{3}=\left(\mu^{2}+\lambda\right) a+\mu \lambda, \\
a^{4}=\left(\mu^{3}+2 \lambda \mu\right) a+\mu^{2} \lambda+\lambda^{2} .
\end{gathered}
$$

Substitution of (11), (12) and (13) in (7) results in

$$
\begin{array}{r}
1 \otimes\left[\left(\mu^{3}+2 \lambda \mu\right) a+\mu^{2} \lambda+\lambda^{2}\right] \\
-4 a \otimes\left[\left(\mu^{2}+\lambda\right) a+\mu \lambda\right]+6(\mu a+\lambda) \otimes(\mu a+\lambda) \\
-4\left[\left(\mu^{2}+\lambda\right) a+\mu \lambda\right] \otimes a \\
+\left[\left(\mu^{3}+2 \lambda \mu\right) a+\mu^{2} \lambda+\lambda^{2}\right] \otimes 1=0 .
\end{array}
$$

Bearing in mind that 1, $a$ are independent, one rearranges the terms of (14) in a systematic way and, in particular, concludes that the "coefficient" of the $a \otimes a$ term must be zero, i.e.,

$$
-4\left(\mu^{2}+\lambda\right)+6 \mu^{2}-4\left(\mu^{2}+\lambda\right)=0
$$


or, upon simplification,

$$
\mu^{2}+4 \lambda=0
$$

Using (11) and (15), we see that

$$
(a-\mu / 2)^{2}=\left(a^{2}-\mu a\right)+\mu^{2} / 4=\lambda-4 \lambda / 4=0 .
$$

Finally, if $\left(a-\lambda_{1}\right)^{2}=\left(a-\lambda_{2}\right)^{2}$ we see that $-2 \lambda_{1} a+\lambda_{1}^{2}=-2 \lambda_{2} a+\lambda_{2}^{2}$, whence $\lambda_{1}=\lambda_{2}$ since $a$ is not central.

Lemma 3.5. Suppose char. $F=3$ and $a, b, c$ are noncentral elements of $B$ such that

$$
\begin{aligned}
\left(a_{r}-a_{l}\right)^{2}\left(b_{r}-b_{l}\right) & =0, \\
\left(a_{r}-a_{l}\right)^{4} & =0, \\
{[a, b]=[a, c] } & =0, \\
{[b, c] } & \neq 0 .
\end{aligned}
$$

Then there exists a unique $\lambda \in F$ such that $(a-\lambda)^{2}=0$.

Proof. Because of char. $F=3$, we note that $\left(a_{r}-a_{l}\right)^{3}=a_{r}^{3}-a_{l}^{3}$ and so $\left(a_{r}-a_{l}\right)^{4}=\left(a_{r}^{3}-a_{l}^{3}\right)\left(a_{r}-a_{l}\right)=0$, which translates under $B_{l} B_{r} \cong B^{o} \times B$ to

$$
1 \otimes a^{4}-a \otimes a^{3}-a^{3} \otimes a+a^{4} \otimes 1=0 .
$$

Since 1, $a$ are independent, Lemma 3.3 applied to (16) says that

$$
a^{3}=\alpha+\beta a, \quad a^{4}=\gamma+\delta a, \quad \alpha, \beta, \gamma, \delta \in F .
$$

But $a^{4}=\alpha a+\beta a^{2}=\gamma+\delta a$, that is,

$$
\beta a^{2}+(\alpha-\delta) a-\gamma=0 .
$$

Suppose $\beta \neq 0$. From $\left(a_{r}-a_{l}\right)^{4}=0$ and (17), we see that for all $y \in B$,

$$
0=[[[[y, a], a], a], a]=\left[\left[y, a^{3}\right], a\right]=\beta[[y, a], a] .
$$

Thus $[[y, a], a]=0$, which implies $a \in F$ by [4, p. 5 Sublemma]. Hence $\beta=0$ and (17) becomes

$$
a^{3}=\alpha .
$$

The given conditions $\left(a_{r}-a_{l}\right)^{2}\left(b_{r}-b_{l}\right)=0$ and $[a, b]=0$ translate to

$$
\left(1 \otimes a^{2}-2 a \otimes a+a^{2} \otimes 1\right)(1 \otimes b-b \otimes 1)=0
$$


or, upon expansion, to

$$
1 \otimes a^{2} b-2 a \otimes a b+a^{2} \otimes b-b \otimes a^{2}+2 a b \otimes a-a^{2} b \otimes 1=0 .
$$

We claim that $1, a, b$ are independent. Indeed, if $b=\alpha+\beta a$, we commute it with $c$ to obtain the contradiction $[b, c]=0$. We can then invoke Lemma 3.3 to conclude in particular from $(20)$ that

$$
a^{2}=\beta+\gamma a+\delta b
$$

We commute (21) with $c$ to get $\delta[b, c]=0$, whence $\delta=0$ and (21) becomes

$$
a^{2}=\beta+\gamma a .
$$

From (22), we deduce that

$$
\begin{gathered}
a^{3}=\gamma \beta+\left(\beta+\gamma^{2}\right) a, \\
a^{4}=\left(\beta^{2}+\gamma^{2} \beta\right)+\left(2 \beta \gamma+\gamma^{3}\right) a .
\end{gathered}
$$

Substitution of (22), (23) and (24) in (16) and subsequent rearrangement of terms result in

$$
\left[2 \beta\left(\beta+\gamma^{2}\right)+\gamma\left(\beta+\gamma^{2}\right) a\right] \otimes 1+[\quad] \otimes a=0 .
$$

This forces

$$
\beta\left(\beta+\gamma^{2}\right)=0, \quad \gamma\left(\beta+\gamma^{2}\right)=0 .
$$

If $\beta=0$, we see from (25) that $\gamma^{3}=0$ whence $\gamma=0$ and (22) becomes $a^{2}=0$. Therefore, without loss of generality, we may assume $\beta \neq 0$ from which we conclude

$$
\beta+\gamma^{2}=0
$$

Finally, form (22), (26) and char. $F=3$, we note that $(a-\gamma / 2)^{2}=\left(a^{2}-\gamma a\right)+$ $\gamma^{4} / 4=\beta+\gamma^{2}=0$ and the proof is complete.

Lemma 3.6. Let $a, b \in B$ such that

$$
\begin{gathered}
a^{2}=b^{2}=[a, b]=0, \\
\left(a_{r}-a_{l}\right)^{2}\left(b_{r}-b_{l}\right)^{2}=0 .
\end{gathered}
$$

Then $a b=0$. 
Proof. (28) translates to

$$
[[[[y, a], a], b], b]=0
$$

for all $y \in B$. Expansion of (29) using (27) leaves us with $-4 a b y a b=0$ for all $y \in B$, whence by the primeness of $B$ we conclude that $a b=0$.

The main idea of the non-PI part of the proof of Theorem 1.1, which occupies Section 5, is to reduce an involution (of the second kind) problem to a noninvolution result. Accordingly, we now state a special case of Howland's Theorem [6, Theorem 3.1]:

Theorem 3.7. Let $R$ and $S$ be centroid simple GPI rings (of char. $\neq 2$ ) which are not PI. Suppose $\alpha:[R, R] \rightarrow[S, S]$ is a Lie isomorphism. Then $\alpha$ can be extended to a map $\sigma: R \rightarrow S$, where $\sigma$ is either an isomorphism or the negative of an antiisomorphism.

Some remarks are in order. The given conditions on $R$ and $S$ are equivalent to saying that $R$ (resp. $S$ ) is a dense ring of finite-rank linear transformations of a vector space $V$ (resp. $W$ ) over a field $E$ (resp. $F$ ), with $V$ and $W$ infinitedimensional. The condition that there exist nonzero orthogonal idempotents $e_{1}, e_{2}$ in $R$ such that $e_{1}+e_{2} \neq 1$ is then easily met. Howland's assumption that $1 \in R$ is not used in his proof. Rather, one can formally set $e_{3}=1-e_{1}-e_{2}$ and use the Pierce decomposition $R=\oplus \sum_{i, j=1}^{3} e_{i} R e_{j}$. It is also important to acknowledge Howland's assumption of char. $\neq 3$. However, this restriction may be removed for the following reason. The assumption of char. $\neq 3$ is only used to establish [6, Lemma 3.6]: if $u \in e_{i} R e_{j}, i \neq j$, then $\left(x^{\alpha}\right)^{2}=0$ (in fact, [6, Lemma 3.6] depends on [6, Lemma 3.3] and it is in the proof of this latter lemma that char. $\neq 3$ is used). But the nature of $R$ in our case is such that given $u \in e_{i} R e_{j}, i \neq j$, one can readily find $v, w \in[R, R]$ such that $u^{2}=v^{2}=w^{2}=u v=v u=u w=w u=0$ but $[v, w] \neq 0$. From these equations one easily establishes that $\left(u_{r}-u_{l}\right)^{4}=\left(u_{r}-u_{l}\right)^{2}\left(v_{r}-v_{l}\right)=$ $0,[u, v]=[u, w]=0$, but $[v, w] \neq 0$. Setting $a=u^{\alpha}, b=v^{\alpha}, c=w^{\alpha}$ and applying $\alpha$ to the preceding relations show us that the hypotheses of Lemma 3.5 are satisfied and accordingly $(a-\lambda)^{2}=0$ for some $\lambda \in F$. This says that $\lambda^{2}=2 \lambda a-a^{2}$ lies in $S$, which forces $\lambda^{2}=0$ (and hence $\lambda=0$ ) since in our situation $S \cap F=0$.

\section{Lie Isomorphisms AND GPI's}

Let $R$ and $S$ be *-prime rings with respective skew elements $K$ and $L$. Let $A=R C_{*} \otimes E$ and $B=S D_{*} \otimes F$ be the respective super ${ }^{*}$-closures of $R$ and 
$S$. We suppose there is a Lie isomorphism

$$
\alpha: \overline{[K, K]} \rightarrow \overline{[L, L]},
$$

where $\overline{[K, K]}=[K, K] /[K, K] \cap C$ and $\overline{[L, L]}=[L, L] /[L, L] \cap D$. The purpose of this section is to show that if $R$ is GPI then $S$ is GPI. We note that $R$ (resp. $S$ ) is GPI if and only if $A$ (resp. $B$ ) is GPI. Also $R$ (resp. $S$ ) is PI if and only if $A$ (resp. $B$ ) is PI. As a first step, by Lemma 2.4 we may extend $\alpha$ to a $\gamma$-semilinear Lie isomorphism

$$
\alpha: \overline{[M, M]} \rightarrow \overline{[N, N]},
$$

where $M$ and $N$ are the respective skew elements of $A$ and $B$.

We make the useful remark that $R$ is PI if and only if $S$ is PI. Indeed, if $R$ is PI then $A$ is PI whence it is well-known that $(M: E)<\infty$ and hence $(\overline{[M, M]}: F)<\infty$. Applying $\alpha$ to $\overline{[M, M]}$ we see that $(\overline{[N, N]}: F)<\infty$, whence it is easy to show that $N$ is PI over $F$. By [8, Theorem 8], $B$ (and hence $S$ ) is PI.

We shall now assume that $R$ is GPI but $S$ is not GPI, and aim at finding a contradiction. As mentioned above, this means that $A$ is GPI and $B$ is not GPI. We divide the argument into two cases.

Case (a): $R$ is of type 2 . Here we let $G \oplus G^{*}$ denote the ${ }^{*}$-socle of $A$. Setting $V=\left\{x-x^{*} \mid x \in[G, G]\right\}$, we note that $\bar{V}$ is the unique minimal Lie ideal of $\overline{[M, M]}$. It follows that $W^{\prime}=\bar{V}^{\alpha}$ must be the unique minimal Lie ideal of $\overline{[N, N]}$. Since $G$ is not PI, we know that $G \cap E=0$ and so we may identify $\bar{V}$ with $V$. Furthermore, there is a natural Lie isomorphism $\nu:[G, G] \rightarrow V$ given by $x \mapsto x-x^{*}$, and so we shall replace $\alpha$ by the composite $\nu \alpha:[G, G] \rightarrow W^{\prime}$. If $S$ is of type 1 , then $W^{\prime}$ is the unique minimal Lie ideal of $\overline{[N, N]}$. If $S$ is of type 2 , then $B$ contains a nonzero *-ideal $T \oplus T^{*}$, and, setting $P=\left\{t-t^{*} \mid t \in[T, T]\right\}$, we note that $\bar{P}$ is a Lie ideal of $\overline{[N, N]}$. Since $W^{\prime}$ is minimal, we see that $W^{\prime} \subseteq \bar{P}$. Letting $U=\left\{u \in[T, T] \mid \overline{u-u^{*}} \in W^{\prime}\right\}$, we see that $U$ is a Lie ideal of $[T, T]$ and that $W^{\prime}=\left\{u-u^{*} \mid u \in U\right\}$. By $[5$, Theorem 5], $U \supseteq[H, H]$ for some nonzero ideal $H$ of $B$ which is contained in $T$ and which is itself a closed prime ring over $F$. By the minimality of $W^{\prime}$, we see that $W^{\prime}=\left\{h-h^{*} \mid h \in[H, H]\right\}$. Using the Lie isomorphism $\mu: W^{\prime} \rightarrow \overline{[H, H]}$ given by $\overline{h-h^{*}} \mapsto \bar{h}$, we may now replace $\nu \alpha$ by the Lie isomorphism $\nu \alpha \mu:[G, G] \rightarrow \overline{[H, H]}$.

Summarizing, we now have a Lie isomorphism (which we continue to label as $\alpha)$ :

$$
\alpha:[G, G] \rightarrow \bar{W},
$$

where either $\bar{W}=W$ is a nonzero Lie ideal of $[N, N]$ if $S$ is of type 1 or $W=[H, H], H$ an ideal of $T$, if $S$ is of type 2 . 
We now begin the construction of a GPI for $B$. Taking any rank-4 idempotent $e$ in $G$ and forming $e G e=M_{4}(E)$, we set $u=e_{12}, v=e_{13}, w=e_{32}$, with $u^{\alpha}=\bar{a}, v^{\alpha}=\bar{b}, w^{\alpha}=\bar{c}, a, b, c \in W$. We first claim $[a, b]=0$. Indeed, writing $e_{14}^{\alpha}=\bar{g}, e_{43}^{\alpha}=\bar{h}, g, h \in W$, we note that $[a, g]$ and $[a, h]$ are central and that $b=[g, h]+\omega, \omega \in F$. Thus $[a, b]=[[a, g], h]+[g,[a, h]]=0$ and similarly $[a, c]=0$. On the other hand, it is clear that $[b, c] \neq 0$ since $[v, w]=u \neq 0$. For all $x \in[G, G]$, one easily checks that

$$
[[[x, u], u], u]=0=[[[x, u], u], v] .
$$

Setting $x^{\alpha}=\bar{y}, y \in W$, applying $\alpha$ to (30), and removing the bars, we find that

$$
[[[y, a], a], a] \in F, \quad[[[y, a], a], b] \in F
$$

for all $y \in W$. Thus $f(X, Y)=[X,[[[Y, a], a], a]] \in B_{F}\langle X, Y\rangle$ is a GPI on $W$ and by $[3$, Remark 9] $f\langle X, Y\rangle=0$. This implies that $[[[Y, a], a], a]=0$ whence $\left(a_{r}-a_{l}\right)^{3}=0$. Similarly one shows that $\left(a_{r}-a_{l}\right)^{2}\left(b_{r}-b_{l}\right)=0$. The conditions of Lemmas 3.4 and 3.5 are now met and we thereby conclude that

$$
(a-\lambda)^{2}=0
$$

for some $\lambda \in F$.

Now let $r=e_{21}$ and set $r^{\alpha}=\bar{d}$. The same argument as in the preceding paragraph results in

$$
(d-\mu)^{2}=0
$$

for some $\mu \in F$. We set $a_{0}=a-\lambda$ and $d_{0}=d-\mu$, noting that $a_{0}^{2}=0=d_{0}^{2}$.

We claim that neither $a_{0} d_{0}=0$ nor $d_{0} a_{0}=0$. Indeed, suppose $d_{0} a_{0}=0$. Applying $\alpha$ to

$$
[[r, u], r]=2 r,
$$

we see that $[[d, a], d]=2 d+\rho, \rho \in F$, i.e.,

$$
\left[\left[d_{0}, a_{0}\right], d_{0}\right]=2 d_{0}+\sigma, \sigma \in F .
$$

But $\left[\left[d_{0}, a_{0}\right], d_{0}\right]=2 d_{0} a_{0} d_{0}=0$, whence the contradiction $2 d_{0}+\sigma=0$.

For all $x \in[G, G]$, one checks that

$$
[[[u, x], u],[[r, y], r]]=\beta e_{11}+\gamma e 22, \beta, \gamma \in E .
$$

It follows that

$$
[[[[u, x], u],[[r, y], r]],[[[u, z], u],[[r, w], r]]]=0
$$


for all $x, y, z, w \in[G, G]$. In $B_{F}\langle X, Y, Z, P, Q\rangle$ (if $S$ is of type 1) or in $H_{F}\langle X, Y, Z, P, Q\rangle$ (if $S$ is of type 2), we set

$$
f(X, Y, Z, P)=\left[\left[\left[\left[a_{0}, X\right], a_{0}\right],\left[\left[d_{0}, Y\right], d_{0}\right]\right],\left[\left[\left[a_{0}, Z\right], a_{0}\right],\left[\left[d_{0}, P\right], d_{0}\right]\right]\right],
$$

which (since $a_{0}^{2}=0=d_{0}^{2}$ ) reduces to

$$
f(X, Y, Z, P)=a_{0} X a_{0} d_{0} Y d_{0} a_{0} Z a_{0} d_{0} P d_{0}+\ldots \ldots \ldots,
$$

where all the terms indicated by the dots have $X, Y, Z, P$ in a different order. An application of $\alpha$ to (32) and removal of the bars show that $[f(X, Y, Z, P), Q]$ is a nontrivial GPI on $W$, a contradiction by [3, Remark 9] since $B$ is not GPI.

Case (b): $R$ is of type 1 . Let $G$ denote the socle of $A$. Here the unique minimal Lie ideal $J$ of $[M, M]$ is $J=[M \cap G, M \cap G]$. Just as in the case (a), we may reduce the problem to the situation where we have a Lie isomorphism (again denoted by $\alpha$ )

$$
\alpha: J \rightarrow \bar{W},
$$

where $\bar{W}=W$ is a nonzero Lie ideal of $[N, N]$ if $S$ is of type 1 or $W=[H, H]$, $H$ an ideal of $T$, if $S$ is of type 2 .

Suppose first that the involution on $G$ is of symplectic type. Pick a symmetric idempotent $e$ of rank 6 and form $e G e=M_{6}(E)$ with the symplectic involution induced on $e G e$ given so that the skew elements of $e G e$ are of the form

$$
\left[\begin{array}{cc}
P & Q \\
U & -P^{t}
\end{array}\right], \quad P, Q, U \quad 3 \times 3 \text { over } E, \quad Q, U \text { symmetric. }
$$

We set $u=e_{14}, v=e_{25}, w=e_{53}+e_{62}$ and set $u^{\alpha}=\bar{a}, v^{\alpha}=\bar{b}, w^{\alpha}=\bar{c}$. We proceed in a similar fashion to the case (a). Using $e_{25}=\left[e_{23}-e_{65}, e_{35}+e_{26}\right]$ one shows that $[a, b]=0$, and using $e_{14}=\left[e_{12}-e_{54}, e_{24}+e_{15}\right]$ one shows that $[a, c]=0$. Clearly, $[b, c] \neq 0$. For all $x \in J$, we have $[[[x, u], u], u]=0=$ $[[[x, u], u], v]$. The proof that $(a-\lambda)^{2}=0$ for some $\lambda \in F$ then goes through as in the case (a). Likewise, setting $r=e_{41}$ (which lies in $J$ as well as does $\left.e_{14}\right)$ and $r^{\alpha}=\bar{d}$, we must conclude that $(d-\mu)^{2}=0$ for some $\mu \in F$. We set $a_{0}=a-\lambda, d_{0}=d-\mu$ and construct the same GPI (33) as in the case (a), thus contradicting the fact that $B$ is not GPI.

We may therefore assume that the involution $\alpha$ on $G$ is of transpose type. We choose a symmetric idempotent $e$ of rank 7 and form $e G e=M_{7}(E)$ so that the involution on $e G e$ is the usual transpose. For $i \neq j$, we set $E_{i j}=e_{i j}-e_{j i}$, where $\left\{e_{i j}\right\}$ are the usual matrix units. We choose $u=E_{12}, v=E_{34}, w=E_{56}$ and set $u^{\alpha}=\bar{a}, v^{\alpha}=\bar{b}, w^{\alpha}=\bar{c}$. The familiar device of writing $u=\left[E_{17}, E_{72}\right]$ can be used to show that $[a, b]=0$ and a similar approach shows that $[a, c]=$ $[b, c]=0$. We next claim that $1, a, b, c$ are $F$-independent. Indeed, suppose

$$
\beta+\gamma a+\delta b+\rho c=0, \quad \beta, \gamma, \delta, \rho \in F .
$$


If, say, $\gamma \neq 0$, we write $E_{27}^{\alpha}=\bar{d}$. A familiar argument shows that $[b, d]=$ $[c, d]=0$ and so commuting (34) with $d$ leaves us with the contradiction $\gamma[a, d]=0$ since $[a, d] \neq 0$. Thus $\gamma$, and similarly $\delta$ and $\rho$, is 0 which then forces $\beta=0$ and establishes our claim. Applying $\alpha$ to

$$
[[[x, u], v], w]=0, \quad x \in J
$$

we obtain

$$
[[[y, a], b], c] \in F
$$

for all $y \in W$. Again using the same argument as in the case (a), we see that the fact that $B$ is not GPI forces

$$
f(X)=[[[X, a], b], c]=0 .
$$

In view of the fact that $a, b, c$ commute with each other, (35) translates to the tensor product equation

$$
1 \otimes a b c-a \otimes b c-b \otimes a c-c \otimes a b+a b \otimes c+a c \otimes b+b c \otimes a-a b c \otimes 1=0 .
$$

An application of Lemma 3.3 to (36) says in particular that

$$
a b=\beta_{0}+\gamma_{0} a+\delta_{0} b+\mu_{0} c, \quad \beta_{0}, \gamma_{0}, \delta_{0}, \mu_{0} \in F .
$$

Setting $E_{67}^{\alpha}=\bar{r}$ and $E_{57}^{\alpha}=\bar{s}, r, s \in W$, we commute (37) with $r$ to obtain

$$
0=\mu_{0}[c, r]=\mu_{0}(s+\rho), \quad \rho \in F,
$$

using the fact that $[a, r]=0=[b, r]$. Therefore $\mu_{0}=0$ and (37) becomes

$$
a b=\beta_{0}+\gamma_{0} a+\delta_{0} b .
$$

We now set $E_{15}^{\alpha}=\bar{g}$ and $E_{52}^{\alpha}=\bar{h}, g, h \in V$, whence $a=[g, h]+\lambda, \lambda \in V \cap F$. Likewise, setting $E_{36}^{\alpha}=\bar{u}$ and $E_{64}^{\alpha}=\bar{v}, u, v \in V$, we have $b=[u, v]+\mu, \mu \in$ $V \cap F$. Analogous to (38), we have the equation

$$
g u=\beta_{1}+\gamma_{1} g+\delta_{1} u, \quad \beta_{1}, \gamma_{1}, \delta_{1} \in F .
$$

Commuting (39) with $h$ yields

$$
[g, h] u=\gamma_{1}[g, h]
$$

and then commuting (40) with $v$ yields

$$
[g, h][u, v]=0,
$$


that is,

$$
(a-\lambda)(b-\mu)=0=(b-\mu)(a-\lambda) .
$$

Setting $a_{0}=a-\lambda$ and $b_{0}=b-\mu$, we see that (41) becomes

$$
a_{0} b_{0}=0=b_{0} a_{0} .
$$

Since $\lambda, \mu \in V \cap F$, we may rewrite $u^{\alpha}$ as $\overline{a_{0}}$ and $v^{\alpha}$ as $\overline{b_{0}}$, with $a_{0} b_{0}=0=b_{0} a_{0}$.

We will need to know that $a_{0}^{2} \neq 0$ and $b_{0}^{2} \neq 0$. Suppose $a_{0}^{2}=0$. We write $\bar{p}=E_{31}^{\alpha}$. In $J$, we have

$$
\left[\left[E_{31}, E_{12}\right], E_{12}\right]=\left[E_{32}, E_{12}\right]=-E_{31} .
$$

An application of $\alpha$ to (42) results in

$$
\left[\left[p, a_{0}\right], a_{0}\right]=-p+\theta, \quad \theta \in V \cap F,
$$

whence

$$
-2 a_{0} p a_{0}=-p+\theta .
$$

Multiplication of (43) on the left and right by $a_{0}$ yields the contradiction $0=-a_{0} p a_{0}$. Therefore we may assume $a_{0}^{2} \neq 0$ and $b_{0}^{2} \neq 0$.

One now checks that

$$
\begin{aligned}
& {\left[\left[\left[E_{12}, x\right], E_{34}\right],\left[\left[E_{12}, y\right], E_{34}\right]\right]=} \\
& E_{12}\left(x E_{34}^{2} y-y E_{34}^{2} x\right) E_{12}+E_{34}\left(x E_{12}^{2} y-y E_{12}^{2} x\right) E_{34}= \\
& \beta E_{12}+\gamma E_{34}
\end{aligned}
$$

for some $\beta, \gamma \in E$. In either $B_{F}\langle X, Y, Z, P, Q\rangle$ (if $S$ is of type 1) or in $H_{F}\langle X, Y, Z, P, Q\rangle$ (if $S$ is of type 2), we define

$$
f(X, Y, Z, P)=\left[\left[\left[\left[a_{0}, X\right], b_{0}\right],\left[\left[a_{0}, Y\right], b_{0}\right]\right],\left[\left[\left[a_{0}, Z\right], b_{0}\right],\left[\left[a_{0}, P\right], b_{0}\right]\right]\right] .
$$

Using $a_{0} b_{0}=b_{0} a_{0}=0$, we see that (45) reduces to

$$
f(X, Y, Z, P)=a_{0} X b_{0}^{2} Y a_{0}^{2} Z b_{0}^{2} P a_{0}+\ldots \ldots,
$$

where all the terms represented in the dots have $X, Y, Z, P$ in a different order. Since $a_{0}^{2} \neq 0$ and $b_{0}^{2} \neq 0$, we see that $f(X, Y, Z, P) \neq 0$ and hence $[f(X, Y, Z, P), Q] \neq 0$. By applying $\alpha$ to (44) we conclude that $[f(X, Y, Z, P), Q]$ is a nontrivial GPI for $V$ and, as we have seen earlier, this forces the contradiction that $B$ is GPI.

Summarizing the results of this section, we have proved

Theorem 4.1. Let $R$ and $S$ be ${ }^{*}$-prime rings with respective skew elements $K$ and $L$, and suppose $\overline{[K, K]}$ is Lie isomorphic to $\overline{[L, L]}$. Then, if either $R$ or $S$ is GPI, both $R$ and $S$ are GPI. 


\section{Involutions of the Second Kind: the non-PI Case.}

We begin this section by proving a special case of Theorem 1.1.

Lemma 5.1. Let $A=G \oplus G^{*}$ and $B=H \oplus H^{*}$ be ${ }^{*}$-simple algebras over algebraically closed fields $E$ and $F$ respectively, with $G$ and $H$ GPI (but not $P I)$ centroid simple of $E$ and $F$ respectively and each equal to its own socle. Let $K=\left\{x-x^{*} \mid x \in G\right\}$ and $L=\left\{y-y^{*} \mid y \in H\right\}$ denote the respective skew elements of $A$ and $B$ and suppose that

$$
\alpha:[K, K] \rightarrow[L, L]
$$

is a $\gamma$-semilinear Lie isomorphism, where $\gamma$ is an isomorphism of $E$ onto $F$. Then there is an isomorphism $\sigma: A \rightarrow B$ such that $\sigma=a$ on $[K, K]$ and such that either $G^{\sigma}=H$ or $G^{\sigma}=H^{*}$.

Proof. Let $\nu:[G, G] \rightarrow[K, K]$ be given by $x \longmapsto x-x^{*}, x \in[G, G]$. It is easy to check that $\nu$ is an $E$-Lie isomorphism. Similarly, $\omega:[H, H] \rightarrow[L, L]$ is an $F$-Lie isomorphism. Then the composite $\delta=\nu \alpha \omega^{-1}$ is a $\gamma$-semilinear Lie isomorphism of $[G, G]$ onto $[H, H]$. By Howland's Theorem (Theorem 3.7), there is a map $\rho: G \rightarrow H$ such that $x^{\rho}=x^{\delta}$ for all $x \in[G, G]$, where either

(a) $\rho$ is an isomorphism

or

(b) $\rho=-\psi, \psi$ an antiisomorphism.

We first consider case (a). We define $\sigma: A \rightarrow B$ according to the rule

$$
\left(x+u^{*}\right)^{\sigma}=x^{\rho}+\left(u^{\rho}\right)^{*}, \quad x, u \in G,
$$

noting that $\left(x^{\sigma}\right)^{*}=\left(x^{\rho}\right)^{*}=\left(x^{*}\right)^{\rho}$. Since

$$
\left(x^{*} u^{*}\right)^{\sigma}=(u x)^{* \sigma}=(u x)^{\rho *}=\left(u^{\rho} x^{\rho}\right)^{*}=\left(x^{\rho}\right)^{*}\left(u^{\rho}\right)^{*}=\left(x^{*}\right)^{\sigma}\left(u^{*}\right)^{\sigma},
$$

we see that $\sigma$ is an isomorphism. Furthermore, for $x \in[G, G], x^{\rho}=x^{\delta}=$ $x^{\nu \alpha \omega^{-1}}$ and so $x^{\rho}=y$, where $\left(x-x^{*}\right)^{\alpha}=y-y^{*}$. Consequently, $\left(x-x^{*}\right)^{\sigma}=$ $x^{\rho}-\left(x^{\rho}\right)^{*}=y-y^{*}=\left(x-x^{*}\right)^{\alpha}$, whence $\sigma=\alpha$ on $[K, K]$.

We look next at case (b), i.e., $\rho=-\psi, \psi$ an antiisomorphism. Defining $\chi: A \rightarrow B$ according to

$$
\left(x+u^{*}\right)^{\chi}=x^{\psi}+\left(u^{\psi}\right)^{*}, \quad x, u \in G,
$$

we see from

$$
(x u)^{\chi}=(x u)^{\psi}=u^{\psi} x^{\psi}=u^{\chi} x^{\chi}
$$


and

$$
\left(x^{*} u^{*}\right)^{\chi}=(u x)^{* \chi}=(u x)^{\psi *}=\left(x^{\psi} u^{\psi}\right)^{*}=u^{\psi *} x^{\psi *}=u^{\chi} x^{\chi}
$$

that $\chi$ is an antiisomorphism. We define $\sigma=* \psi$ and see immediately that $\sigma$ is an isomorphism of $A$ onto $B$. Since $x^{\rho}=y, x \in[G, G]$, where $\left(x-x^{*}\right)^{\alpha}=$ $y-y^{*}$, we conclude from

$$
\left(x-x^{*}\right)^{\sigma}=\left(x-x^{*}\right)^{* \chi}=-\left(x-x^{*}\right)^{\chi}=-x^{\chi}+\left(x^{\chi}\right)^{*}=x^{\rho}-\left(x^{\rho}\right)^{*}=y-y^{*}
$$

that $\sigma=\alpha$ on $[K, K]$. The proof of the lemma is now complete.

We next generalize Lemma 5.1 to

Theorem 5.2. Let $A$ and $B$ be ${ }^{*}$-closed ${ }^{*}$-prime algebras with involutions of the second kind over algebraically closed fields $E$ and $F$ respectively, and assume that $A$ and $B$ are GPI but not PI. Let $K$ and $L$ denote the respective skew elements of $A$ and $B$ and suppose that

$$
\alpha: \overline{[K, K]} \rightarrow \overline{[L, L]}
$$

is a $\gamma$-semilinear Lie isomorphism, where $\gamma: E \rightarrow F$ is an isomorphism. Then there is a $\gamma$-semilinear ring monomorphism

$$
\psi:\langle[K, K]\rangle \rightarrow\langle[L, L]\rangle C_{B}+C_{B}
$$

such that

$$
x^{\psi}=y+\rho, x \in[K, K], y \in[L, L], \bar{y}=\bar{x}^{\alpha}, \rho \in L \cap C_{B},
$$

where $C_{B}$ is the extended centroid of $B$.

Proof. With reference to the discussion in Section 1, we let $A_{0}=G \oplus G^{*}$ and $B_{0}=H \oplus H^{*}$ be the respective ${ }^{*}$-socles of $A$ and $B$. Setting $V=$ $\left\{x-x^{*} \mid x \in[G, G]\right\}$ and $W=\left\{y-y^{*} \mid y \in[H, H]\right\}$, we know that $\bar{V}$ and $\bar{W}$ are the unique minimal Lie ideals of $[K, K]$ and $\overline{[L, L]}$ respectively. Therefore $\alpha$ induces a $\gamma$-semilinear Lie isomorphism of $\bar{V}$ onto $\bar{W}$. Furthermore, since neither $G$ nor $H$ is PI, we know that neither $V$ nor $W$ contains nonzero central elements, that is, we may write $\bar{V}=V$ and $\bar{W}=W$. The conditions of Lemma 5.1 (applied to $A_{0}$ and $B_{0}$ ) are now fulfilled and accordingly there is an isomorphism $\sigma: A_{0} \rightarrow B_{0}$ such that $\sigma=\alpha$ on $V$ where (by interchanging $H$ and $H^{*}$ if necessary) we may assume that $G^{\sigma}=H$ and $\left(G^{*}\right)^{\sigma}=H^{*}$. We now embark upon a series of claims in order to complete the proof of the theorem.

For Claims 1 through 6 , we fix $x \in[K, K]$ and $y \in[L, L]$ such that $\bar{y}=\bar{x}^{\alpha}$. 
Claim 1. For $u \in V,[x, u]^{\sigma}=\left[y, u^{\sigma}\right]$.

Proof. $[x, u]^{\sigma}=\overline{[x, u]^{\sigma}}=\overline{[x, u]}^{\alpha}=\left[\bar{x}^{\alpha}, \bar{u}^{\alpha}\right]=\left[\bar{y}, \overline{\left.u^{\sigma}\right]}=\overline{\left[y, u^{\sigma}\right]}=\left[y, u^{\sigma}\right]\right.$.

Claim 2. For $u \in V,\left[(x u)^{\sigma}-\left[y u^{\sigma}, u^{\sigma}\right]=0\right.$

Proof. $\left.\left[(x u)^{\sigma}-y u^{\sigma}, u^{\sigma}\right]=[x u, u]^{\sigma}-y u^{\sigma}, u^{\sigma}\right]=([x, u] u)^{\sigma}-\left[y, u^{\sigma}\right] u^{\sigma}=$ $\left([x, u]^{\sigma}-\left[y, u^{\sigma}\right]\right) u^{\sigma}=0$ by Claim 1 .

A linearization of Claim 2 yields

Claim 3. For $u, v \in V,\left[(x u)^{\sigma}-y u^{\sigma}, v^{\sigma}\right]+\left[(x v)^{\sigma}-y v^{\sigma}, u^{\sigma}\right]=0$.

Claim 4. Let $u, v \in V, a=u^{\sigma}$, and $b=(x u)^{\sigma}-y u^{\sigma}$. Then $a r b=b r a$ for all $r \in W$.

Proof. Since $([x, v] u)^{\sigma}=[x, v]^{\sigma} u^{\sigma}$, we have

$$
\begin{aligned}
0= & {\left[([x, v] u)^{\sigma}-[x, v]^{\sigma} u^{\sigma}, u^{\sigma}\right] } \\
& {\left[(x v u-v x u)^{\sigma}-\left[y, v^{\sigma}\right] u^{\sigma}, u^{\sigma}\right] \quad(\text { Claim 1) }} \\
& {\left[\left((x v)^{\sigma}-y v^{\sigma}\right) u^{\sigma}-v^{\sigma}\left((x u)^{\sigma}-y u^{\sigma}\right), u^{\sigma}\right] } \\
& {\left[(x v)^{\sigma}-y v^{\sigma}, u^{\sigma}\right] u^{\sigma}-\left[v^{\sigma}, u^{\sigma}\right]\left((x u)^{\sigma}-y u^{\sigma}\right) \quad \text { (Claim 2) } } \\
& -\left[(x u)^{\sigma}-y u^{\sigma}, v^{\sigma}\right] u^{\sigma}-\left[v^{\sigma}, u^{\sigma}\right]\left((x u)^{\sigma}-y u^{\sigma}\right) \quad \text { (Claim 3). }
\end{aligned}
$$

Since $\sigma=\alpha$ on $\nu$ and $\alpha: V \rightarrow W$ is surjective, we set $r=v^{\sigma}$ and see that (46) becomes

$$
-[b, r] a-[r, a] b=0
$$

for all $r \in W$. In turn, (47) reduces to arb $=b r a, r \in W$, and the claim is established.

At this point we recall that $C_{B}=F e \oplus F e^{*}$, with $C_{H}=F e, e+e^{*}=$ $1, e e^{*}=0$.

Claim 5. Let $a$ and $b$ be as in Claim 4. Then $b$ is a symmetric element and $b=\rho a$, where $\rho=\rho_{x, y, u}$ is a skew element of $C_{B}$.

Proof. Since ${ }^{*}$ commutes with $\sigma$, it is easy to check that $b^{*}=(u x)^{\sigma}-u^{\sigma} y$ and so by Claim 1 we see that $b=b^{*}$. We now write $a=a_{1}-a_{1}^{*}, a_{1} \in H$ (since $a \in W), b=b_{1}+b_{1}^{*}, b_{1} \in H$, and $r=h-h^{*}, h \in[H, H]$. Then Claim 4 yields

$$
a_{1} h b_{1}=b_{1} h a_{1}, \quad h \in[H, H] .
$$

By [3, Theorem 4.3.11], we may pick an idempotent $g \in H$ such that $a_{1}, b_{1} \in$ $g H g$ and $g \notin[H, H]$. By Theorem 3.1,H=[H,H]+Fg and thus $b_{1}=$ 
$\alpha e a_{1}, \lambda \in F$. Thus $b_{1}^{*}=\lambda e^{*} a_{1}^{*}$ whence $b=\lambda e a_{1}+\lambda e^{*} a_{1}^{*}=\lambda\left(e-e^{*}\right)\left(a_{1}-a_{1}^{*}\right)$. Hence $b=\rho a$, where $\rho=\lambda\left(e-e^{*}\right)$ is a skew element of $C_{B}$.

Claim 6. The element $\rho=\rho_{x, y, u}$ is independent of the choice of $u \in V$, that is, $\rho=\rho_{x, y}$.

Proof. Let $u_{1}, u_{2} \in V$. Making repeated use of Claim 5, we may write

$$
\begin{aligned}
\left(x u_{1}\right)^{\sigma}-y u_{1}^{\sigma} & =\rho_{1} u_{1}^{\sigma}, \\
\left(x u_{2}\right)^{\sigma}-y u_{2}^{\sigma} & =\rho_{2} u_{2}^{\sigma}, \\
\left(x\left(u_{1}+u_{2}\right)\right)^{\sigma}-y\left(u_{1}+u_{2}\right)^{\sigma} & =\rho_{3}\left(u_{1}+u_{2}\right)^{\sigma},
\end{aligned}
$$

where $\rho_{1}, \rho_{2}, \rho_{3}$ are skew elements in $C_{B}$. Expanding and simplifying the equations (48), we are left with

$$
\left(\rho_{1}-\rho_{3}\right) u_{1}^{\sigma}+\left(\rho_{2}-\rho_{3}\right) u_{2}^{\sigma}=0 .
$$

We set $u_{1}^{\sigma}=a_{1}-a_{1}^{*}, u_{2}^{\sigma}=a_{2}-a_{2}^{*}, a_{1}, a_{2} \in H, \rho_{1}-\rho_{3}=\lambda_{1}\left(e-e^{*}\right), \rho_{2}-$ $\rho_{3}=\lambda_{2}\left(e-e^{*}\right), \lambda_{1}, \lambda_{2} \in F$. Then (49) becomes

$$
\lambda_{1}\left(e-e^{*}\right)\left(a_{1}-a_{1}^{*}\right)+\lambda_{2}\left(e-e^{*}\right)\left(a_{2}-a_{2}^{*}\right)=0,
$$

whence in particular

$$
\left(\lambda_{1} e\right) a_{1}+\left(\lambda_{2} e\right) a_{2}=0 .
$$

If $a_{1}, a_{2}$ are $F e$-independent, we see from (50) that

$$
\lambda_{1} e=0=\lambda_{2} e .
$$

An application of $*$ to $(51)$ results in $\rho_{1}-\rho_{3}=0=\rho_{2}-\rho_{3}$, i.e., $\rho_{1}=\rho_{3}=\rho_{2}$. If $a_{1}, a_{2}$ are $F e$-dependent, then one may choose $u_{3} \in V$ such that $a_{1}, a_{2}$ are $F e$-independent, where $u_{3}^{\sigma}=a_{3}-a_{3}^{*}$.. Writing $\left(x u_{3}\right)^{\sigma}-y u_{3}^{\sigma}=\lambda_{3} u_{3}^{\sigma}$, we see from the preceding argument that $\rho_{1}=\lambda_{3}=\rho_{2}$ and the proof is complete.

In view of Claim 6 , we may now write

$$
(x u)^{\sigma}-y u^{\sigma}=\rho_{x, y} u^{\sigma}
$$

for all $x \in[K, K], y \in[L, L]$ such that $\bar{y}=\bar{x}^{\alpha}, u \in V$. We rewrite (52) as

$$
(x u)^{\sigma}=t_{x, y} u^{\sigma}, \quad t_{x, y}=y+\rho_{x, y} .
$$

Claim 7. $t_{x, y}$ is independent of $y$, i.e., $t_{x, y}=t_{x}$. 
Proof. If $(x u)^{\sigma}=t_{x, y_{1}} u^{\sigma}=t_{x, y_{2}} u^{\sigma}$, then $\left(t_{x, y_{1}}-t_{x, y_{2}}\right) W=0$ whence $\left(t_{x, y_{1}}-t_{x, y_{2}}\right)\langle W\rangle=0$. But $\langle W\rangle$ contains a nonzero *-ideal $I$ of $B$, which forces $t_{x, y_{1}}=t_{x, y_{2}}$.

In view of Claim 7 , we define $\phi:[K, K] \rightarrow[L, L] C_{B}+C_{B}$ according to $x^{\phi}=t_{x}$, noting that

$$
(x u)^{\sigma}=x^{\phi} u^{\sigma}, \quad x \in[K, K], u \in V .
$$

Claim 8. $(x u)^{\sigma}=u^{\sigma} x^{\phi}, \quad x \in[K, K], u \in V$.

Proof. Analogous to equation (52), one has

$$
(u x)^{\sigma}-u^{\sigma} y=\mu_{x, y} u^{\sigma}, \quad \mu_{x, y} \text { skew in } C_{B},
$$

for $u \in V, x \in[K, K], y \in[L, L]$ such that $\bar{y}=\bar{x}^{\alpha}$. Using Claim 1, we subtract (53) from (52) to obtain

$$
0=[x, u]^{\sigma}-\left[y, u^{\sigma}\right]=\left(\rho_{x, y}-\mu_{x, y}\right) u^{\sigma} .
$$

Since nonzero skew elements in $C_{B}$ are invertible, we must conclude that $\mu_{x, y}=\rho_{x, y}$, and the claim is proved.

Claim 9. Let $x_{1}, x_{2}, \ldots, x_{k} \in[K, K]$ and $u, v \in V$. Then $u^{\sigma} x_{1}^{\phi} x_{2}^{\phi} \ldots x_{k}^{\phi} v^{\sigma}=$ $\left(u x_{1} x_{2} \ldots x_{k} v\right)^{\sigma}$.

Proof. The proof is by induction on $k$. For $k=1$, we have $u^{\sigma} x_{1}^{\phi} v^{\sigma}=$ $\left(u x_{1}\right)^{\sigma} v^{\sigma}=\left(u x_{1} v\right)^{\sigma}$. Next, assuming the result is true for $k-1$, we have

$$
\begin{aligned}
u^{\sigma} x_{1}^{\phi} \ldots x_{k}^{\phi} v^{\sigma} & = \\
\left(u x_{1}\right)^{\sigma} x_{2}^{\phi} \ldots x_{k}^{\phi} v^{\sigma} & = \\
{\left[u, x_{1}\right]^{\sigma} x_{2}^{\phi} \ldots x_{k}^{\phi} v^{\sigma}+\left(x_{1} u\right)^{\sigma} x_{2}^{\phi} \ldots x_{k}^{\phi} v^{\sigma} } & = \\
\left(\left[u, x_{1}\right] x_{2} \ldots x_{k} v\right)^{\sigma}+x_{1}^{\phi} u^{\sigma} x_{2}^{\phi} \ldots x_{k}^{\phi} v^{\sigma} & = \\
\left(u x_{1} x_{2} \ldots x_{k} v\right)^{\sigma}-\left(x_{1} u x_{2} \ldots x_{k} v\right)^{\sigma}+x_{1}^{\phi}\left(u x_{2} \ldots x_{k} v\right)^{\sigma} & = \\
\left(u x_{1} x_{2} \ldots x_{k} v\right)^{\sigma}-\left(x_{1} u x_{2} \ldots x_{k} v\right)^{\sigma}+x_{1}^{\phi} u^{\sigma}\left(x_{2} \ldots x_{k} v\right)^{\sigma} & = \\
\left(u x_{1} \ldots x_{k} v\right)^{\sigma}-\left(x_{1} u x_{2} \ldots x_{k} v\right)^{\sigma}+\left(x_{1} u\right)^{\sigma}\left(x_{2} \ldots x_{k} v\right)^{\sigma} & = \\
\left(u x_{1} \ldots x_{k} v\right)^{\sigma}-\left(x_{1} u x_{2} \ldots x_{k} v\right)^{\sigma}+\left(x_{1} u x_{2} \ldots x_{k} v\right)^{\sigma} & = \\
\left(u x_{1} \ldots x_{k} v\right)^{\sigma} . &
\end{aligned}
$$


We now define $\psi:\langle[K, K]\rangle \rightarrow\langle[L, L]\rangle C_{B}+C_{B}$ via

$$
\sum x_{1} x_{2} \ldots x_{k} \longmapsto \sum x_{1}^{\phi} x_{2}^{\phi} \ldots x_{k}^{\phi}, \quad x_{i} \in[K, K] .
$$

To show $\psi$ is well-defined, suppose $\sum x_{1} x_{2} \ldots x_{k}=0$. Then by Claim 9, $u^{\sigma}\left(\sum x_{1}^{\phi} \ldots x_{k}^{\phi}\right) v^{\sigma}=\left(\sum u x_{1} \ldots x_{k} v\right)^{\sigma}=\left\{u\left(\sum x_{1} x_{2} \ldots x_{k}\right) v\right\}^{\sigma}=0$ for all $u, v \in V$, whence $\sum x_{1}^{\phi} x_{2}^{\phi} \ldots x_{k}^{\phi}=0$. Clearly, $\psi$ is a $\gamma$-semilinear ring homomorphism by the nature of its definition. To show $\psi$ is injective, suppose $J=\operatorname{ker} \psi \neq 0$. Since $J$ is a nonzero ${ }^{*}$-ideal of $A, J \cap V=V$ (since $V=\bar{V}$ is the unique minimal Lie ideal of $\overline{[K, K]}$ ). To reach a contradiction, it suffices to show that $\psi=\alpha$ on $V$ since $\alpha$ is an injection. Indeed, for $u, v \in V$, we note that $u^{\sigma} v^{\sigma}=(u v)^{\sigma}=u^{\phi} v^{\sigma}$. Thus $\left(u^{\sigma}-u^{\phi}\right) W=0$, whence $u^{\sigma}=u^{\phi}$. But then $u^{\psi}=u^{\phi}=u^{\sigma}=u^{\alpha}$. Finally, from $x^{\psi}=x^{\phi}=t_{x}$ we have

$$
x^{\psi}=y+\rho_{x, y}, y \in[L, L], \bar{y}=\bar{x}^{\alpha}, \rho_{x, y} \text { skew in } C_{B},
$$

and the proof of Theorem 5.2 is thereby complete.

\section{Involutions of the Second Kind: the PI Case}

The aim of this section is to prove Theorem 1.1 for the special case (Theorem 6.2) where $R$ and $S$ are their own super ${ }^{*}$-closures and furthermore are PI. The proof of Theorem 6.2 rests heavily upon the following noninvolution result on Lie isomorphisms.

Theorem 6.1. Let $G=M_{n}(E), H=M_{n}(F), E$ and $F$ fields with an isomorphism $\gamma: E \rightarrow F$. Assume $n>2$ if char. $E \neq 3$ and $n>3$ if char. $E=3$. Suppose there is a $\gamma$-semilinear Lie isomorphism

$$
\alpha: \overline{[G, G]} \rightarrow \overline{[H, H]}
$$

where $\overline{[G, G]}=[G, G] /[G, G] \cap E$ and $\overline{[H, H]}=[H, H] /[H, H] \cap F$. Then $n=m$ and there exists a $\gamma$-semilinear map $\sigma: G \rightarrow H$ such that $\sigma$ is either an isomorphism or the negative of an antiisomorphism and such that $\overline{x^{\sigma}}=\bar{x}^{\alpha}$ for all $x \in[G, G]$.

Proof. The fact that $\operatorname{dim}_{E} \overline{[G, G]}=\operatorname{dim}_{F} \overline{[H, H]}$ says not only that $n=m$ but that $[G, G] \cap E=0$ if and only if $[H, H] \cap F=0$. If $[G, G] \cap E=0$, then our result is a direct consequence of Howland's Theorem since the condition $n>2$ assures the presence of three orthogonal idempotents. Therefore, for the remainder of the proof, we assume $[G, G] \cap E \neq 0$ (and hence $[H, H] \cap F \neq 0$ ), which forces $E$ to have characteristic $p$ with $p$ dividing $n$. If $p \neq 3$, we have 
$p \geq 5$ which forces $n \geq 5$. If $p=3$, then the given condition $n>3$ forces $n \geq 6$. Let $\left\{e_{i j} \mid i, j=1,2, \ldots, n\right\}$ denote the usual matrix units for $G$. The proof will largely involve establishing a series of claims.

Claim 1. If $\left[e_{i j}, e_{k l}\right]=0, i \neq j, k \neq l$, and ${\overline{e_{i j}}}^{\alpha}=\bar{a},{\overline{e_{k l}}}^{\alpha}=\bar{b}, a, b \in$ $[H, H]$, then $[a, b]=0$.

Proof. Without loss of generality the two cases to consider are (a) $e_{12}, e_{13}$ and (b) $e_{12}, e_{34}$. In (a), write ${\overline{e_{14}}}^{\alpha}=\bar{c}$ and ${\overline{e_{43}}}^{\alpha}=\bar{d}$. From $\left[e_{12}, e_{13}\right]=$ $\left[e_{12}, e_{14}\right]=\left[e_{12}, e_{43}\right]=0$ and $e_{13}=\left[e_{14}, e_{43}\right]$, we obtain $[a, c],[a, d], b-[c, d]$ are central, whence it is easy to see that $[a, b]=0$. Similarly for (b), since $n \geq 5$, we write $e_{34}=\left[e_{35}, e_{54}\right]$ to arrive at $[a, b]=0$.

Claim 2. For $i \neq j,{\overline{e_{i j}}}^{\alpha}=\overline{a_{i j}}, a_{i j}$ is unique such that $a_{i j} \in[H, H]$ and $a_{i j}^{2}=0$.

Proof. Without loss of generality we may consider $u=e_{12}$, and, setting $v=$ $e_{13}$ and $w=e_{32}$, we write $\bar{u}^{\alpha}=\bar{a}, \bar{v}^{\alpha}=\bar{b}, \bar{w}^{\alpha}=\bar{c}, a, b, c \in[H, H]$. By Claim $1,[a, b]=0=[a, c]$, but clearly $[b, c] \neq 0$. From $[[[x, u], u], u]=0$ for all $x \in$ $[G, G]$, it is easy to see that $[[[y, a], a], a]=\lambda \in F$ for all $y \in[H, H]$. By Lemma 3.2 , pick $s \in[H, H]$ such that $s a \notin[H, H]$. One checks that $[[[s a, a], a], a]=$ $[[[s, a], a], a] a=\mu a$ for some $\mu \in F$ and so we have $[[[y, a], a], a] \in F+F a$ for all $y \in H$. Commuting with $a$ then yields $[[[[y, a], a], a], a]=0$ for all $y \in H$, i.e.,

$$
\left(a_{r}-a_{l}\right)^{4}=0
$$

From $[[[x, u], u], v]=0$ for all $x \in[G, G]$, we conclude that $[[[y, a], a], b] \in F$ for all $y \in[H, H]$. By Lemma 3.2, pick $s \in[H, H]$ such that as $\notin[H, H]$ which, as we have seen earlier, leads to [[[y,a], a],b] $F+F b$. Since $a$ and $b$ are clearly $F$ independent modulo $F$, we must have $[[[y, a], a], b] \in F$ for all $y \in H$. Suppose for some $y,[[[y, a], a], b]=\lambda \neq 0$. Then $[[[y a, a], a], b]=[[[y, a], a], b] a=\lambda a=$ $\mu$, whence the contradiction $a \in F$. Thus $[[[y, a], a], b]=0$ for all $y \in H$, i.e.,

$$
\left(a_{r}-a_{l}\right)^{2}\left(b_{r}-b_{l}\right)=0 .
$$

The conditions of Lemma 3.5 (and hence of Lemma 3.4) are now fulfilled, so we conclude that $(a-\lambda)^{2}=0$, where $\lambda \in F$ is unique. But $F \subseteq[H, H]$, so $a$ may be replaced by $a_{12}=a-\lambda \in[H, H]$ with $a_{12}^{2}=0$.

Claim 3. Let $i, j, k$ be distinct. Then either

(a) $a_{i j} a_{j k}=a_{i k}, \quad a_{j k} a_{i j}=0$ or

(b) $a_{i k}=-a_{j k} a_{i j}, \quad a_{i j} a_{j k}=0$. 
Proof. For simplicity, we let $i=1, j=2, k=3$, set $u=e_{12}, v=e_{23}, w=$ $e_{13}$, and write $a=a_{12}, b=a_{23}, c=a_{13}$. By Claim $2, a^{2}=b^{2}=c^{2}=0$. From $[[[x, u], u], w]=0$ for all $x \in[G, G]$, we are led by a familiar argument to $[[[[y, a], a], c], c]=0$ for all $y \in H$, i.e., $\left(a_{r}-a_{l}\right)^{2}\left(c_{r}-c_{l}\right)^{2}=0$. By Lemma $3.6, a c=0=c a$, and, in a similar fashion, we have $b c=0=c b$. From $[[[[x, u], u], v], v]=0$ for all $x \in[G, G]$, we obtain

$$
[[[[y, a], a], b], b]=\lambda \in F, \quad y \in[H, H] .
$$

Since $a^{2}=b^{2}=0,(54)$ reduces to

$$
\text { bayab }=\lambda \in F, \quad y \in[H, H] .
$$

Since $b c=0$, multiplication of (55) by $c$ leaves us with $\lambda c=0$, whence $\lambda=0$ and (55) becomes

$$
\text { bayab }=0, \quad y \in[H, H] .
$$

By Lemma 3.2, there exists $s \in[H, H]$ such that $s a \notin[H, H]$. Now $b a(s a) a b=$ 0 since $a^{2}=0$, and so (55) becomes

$$
b a y a b=0, \quad y \in H .
$$

Since $H$ is prime, either $a b=0$ or $b a=0$. From $[u, v]=w$ we conclude that

$$
[a, b]=c+\mu, \quad \mu \in F
$$

Multiplication of (57) by $c$ results in $\mu c=0$ which forces $\mu=0$, that is, $a b-b a=c$. If $b a=0$ then $a b=c$, and if $a b=0$, then $c=-b a$.

Claim 4. For $i, j, k, l$ distinct, $a_{i j}=a_{i k} a_{k j}$ if and only if $a_{i j}=a_{i l} a_{l j}$.

Proof. Suppose $a_{i j}=a_{i k} a_{k j}$ but (see Claim 3) $a_{i j}=-a_{l j} a_{i l}$. By Claim 3 , either (i) $a_{k j}=a_{k i} a_{i j}$ or (ii) $a_{k j}=-a_{i j} a_{k i}$. In case (i), $a_{k j}=a_{k i} a_{i j}=$ $-a_{k i} a_{l j} a_{i l}=0$ since $a_{k i} a_{l j}=0$ by Claim 3. In case (ii), $a_{k j}=-a_{i j} a_{k i}=$ $-a_{i k} a_{k j} a_{k i}=0$ since $a_{k j} a_{k i}=0$. In either case, a contradiction is reached.

For temporary purposes, we shall say that $a_{i j}, i \neq j$, is good if there exists $k \neq i, j$ such that $a_{i j}=a_{i k} a_{k j}$. In view of Claim 4 , we know that $a_{i j}, i \neq j$, is good if and only if $a_{i j}=a_{i k} a_{k j}$ for all $k \neq i, j$.

Claim 5. If $a_{i j}$ is good for some $i \neq j$, then $a_{k l}$ is $\operatorname{good}$ for all $k \neq l$.

Proof. We may suppose that $a_{12}$ is good. We first show that $a_{1 j}$ is good for $j \neq 1,2$. If $a_{12}$ is not good, then by Claim $3, a_{1 j}=-a_{k j} a_{1 k}$ for some 
$k \neq 1, j$. We then have the contradiction $a_{12}=a_{1 j} a_{j 2}=-a_{k j} a_{1 k} a_{j 2}=0$ since $a_{1 k} a_{j 2}=0$. By a symmetric argument applied to $a_{1 j}, j \neq 1$, we conclude that $a_{i j}$ is good for all $i \neq j$ where $j \neq 1$. It only remains to show that $a_{i 1}$ is good for all $i \neq 1$. Pick $k \neq 1, j$ and note that $a_{i k}$ is good. Then a symmetric argument to the above one shows that $a_{i 1}$ is good and the claim is established.

At this point we assume that some $a_{i j}, i \neq j$, is good. In view of Claim 4 , this says that all $a_{i j}, i \neq j$, are good. From this it follows that, for $i, k, l$ distinct, $a_{i k} a_{k i}=a_{i k}\left(a_{k l} a_{l i}\right)=\left(a_{i k} a_{k l}\right) a_{l i}=a_{i l} a_{l i}$. This enables us to define $a_{i i}=a_{i k} a_{k i}$, where $k$ is any subscript distinct from $i$.

Claim 6. $\left\{a_{i j} \mid i, j=1,2, \ldots, n\right\}$ is a set of matrix units for $H$.

Proof. One simply examines all possible cases as follows (parentheses will indicate products which are zero by Claim 3):

$$
\begin{aligned}
a_{12} a_{23} & =a_{13}, \\
a_{12} a_{21} & =a_{11}, \\
a_{12} a_{22}=a_{12} a_{23} a_{32} & =a_{13} a_{32}=a_{12}, \\
a_{12} a_{33} & =\left(a_{12} a_{31}\right) a_{13}=0, \\
a_{12} a_{34} & =a_{14}\left(a_{42} a_{34}\right)=0, \\
\left(a_{12} a_{31}\right) & =0, \\
a_{12} a_{32} & =\left(a_{12} a_{31}\right) a_{12}=0, \\
a_{11} a_{11}=a_{12} a_{21} a_{13} a_{31} & =a_{12} a_{23} a_{31}=a_{12} a_{21}=a_{11}, \\
a_{11} a_{22} & =a_{13}\left(a_{31} a_{23}\right) a_{32}=0 .
\end{aligned}
$$

All essentially distinct cases having been considered, the proof of Claim 6 is complete.

We may now complete the proof of Theorem 6.1 in the case that all the $a_{i j}$ 's are good. One simply defines $\sigma: G \rightarrow H$ according to $e_{i j}^{\sigma}=a_{i j}, i, j=1,2, \ldots, n$ and its extension by $\gamma$-semilinearity. Clearly, $\overline{e_{i j}^{\sigma}}={\overline{a_{i j}}}={\overline{e_{i j}}}^{\alpha}$ for $i \neq j$, and, in view of $\left(e_{i i}-e_{j j}\right)^{\sigma}=\left[e_{i j}, e_{j i}\right]^{\sigma}=\left[a_{i j}, a_{j i}\right]$, we have $\overline{\left(e_{i i}-e_{j j}\right)^{\sigma}}=\left[\overline{a_{i j}}, \overline{a_{j i}}\right]=$ $\left[{\overline{e_{i j}}}^{\alpha},{\overline{e_{j i}}}^{\alpha}\right]={\overline{e_{i i}-e_{j j}}}^{\alpha}$. Thus $\overline{x^{\sigma}}=\bar{x}^{\alpha}$ for all $x \in[G, G]$.

It remains now to consider the situation where none of the $a_{i j}, i \neq j$, are good, i.e., for all $i, j, k$ distinct,

$$
a_{i j} a_{j k}=0, \quad a_{i k}=-a_{j k} a_{i j} .
$$

Let $*$ be the transpose map in $G$. Clearly, $*$ is an $E$-linear antiautomorphism of $G$ such that -* induces an $E$-linear Lie automorphism of $\overline{[G, G]}$. Then 
$\beta=-* \alpha$ is a $\gamma$-semilinear Lie isomorphism of $\overline{[G, G]}$ onto $\overline{[H, H]}$. From the definition of $\beta$, it is straightforward to verify that the unique element $b_{i j} \in[H, H], b_{i j}^{2}=0$ such that ${\overline{e_{i j}}}^{\beta}=\overline{b_{i j}}, i \neq j$, is $b_{i j}=-a_{j i}$. Furthermore, for $i, j, k$ distinct, we see from

$$
b_{i j} b_{j k}=\left(-a_{j i}\right)\left(-a_{k j}\right)=a_{j i} a_{k j}=-a_{k i}=b_{i k}
$$

that all $b_{i j}, i \neq j$, are good (relative to $\beta$ ). By the first part of the proof we then know that there is an isomorphism $\chi: G \rightarrow H$ such that $\overline{x^{\chi}}=\bar{x}^{\beta}=-{\overline{x^{*}}}^{\alpha}$ for all $x \in[G, G]$. Then $\sigma=-* \chi$, being the negative of the antiisomorphism $* \chi$, is the required map, and the proof of Theorem 6.1 is now complete.

Theorem 6.2. Let $A$ and $B$ be ${ }^{*}$-closed ${ }^{*}$-prime algebras of type 2 over respective algebraically closed fields $E$ and $F$, let $C$ and $D$ be the respective extended centroids of $A$ and $B$, and let $K$ and $L$ be the respective skew elements of $A$ and $B$. Assume that $A$ is $P I$ and that $A$ does not satisfy $S_{4}$ (if char. $\neq 3$ ) or $S_{6}($ if char. $=3)$. We set $\overline{[K, K]}=[K, K] /[K, K] \cap C, \overline{[L, L]}=[L, L] \cap D$, and suppose there is a $\gamma$-semilinear Lie isomorphism $\alpha: \overline{[K, K]} \rightarrow \overline{[L, L]}$, where $\gamma: E \rightarrow F$ is an isomorphism. Then there exists a $\gamma$-semilinear isomorphism $\psi: A \rightarrow B$ such that for all $x \in[K, K], x^{\psi}=y+\rho, y \in[L, L]$, $\bar{y}=\bar{x}^{\alpha}, \rho \in[L, L] \cap D$.

Proof. From the given conditions we see that $E=C_{*}, F=D_{*}$, and $B$ is also PI. We have $A=G \oplus G^{*}, B=H \oplus H^{*}$, with $G=M_{n}(E), H=M_{m}(F)$. Clearly, $K=\left\{x-x^{*} \mid x \in G\right\}$ and $L=\left\{y-y^{*} \mid y \in H\right\}$. The composite $\delta: \overline{[G, G]} \rightarrow \overline{[H, H]}$ given by

$$
\bar{x} \stackrel{\nu}{\longmapsto} \overline{x-x^{*}} \stackrel{\alpha}{\longmapsto} \overline{y-y^{*}} \stackrel{\omega^{-1}}{\longmapsto} \bar{y}
$$

is a $\gamma$-semilinear Lie isomorphism. By Theorem $6.1, n=m$ and there exists a $\gamma$-semilinear map $\sigma: G \rightarrow H$ such that $\overline{x^{\sigma}}=\bar{x}^{\delta}$ for all $x \in[G, G]$, where either (a) $\sigma$ is an isomorphism or (b) $\sigma=-\theta, \theta$ an antiisomorphism. We sketch the remainder of the proof, following almost verbatim the relevant part of the proof of Theorem 5.1. In case (a), we define $\psi: A \rightarrow B$ via $\left(x+u^{*}\right)^{\psi}=x^{\sigma}+\left(u^{\sigma}\right)^{*}, x, u \in G$. In case (b), we define an antiautomorphism $\chi: A \rightarrow B$ via $\left(x+u^{*}\right)^{\chi}=x^{\theta}+\left(u^{\theta}\right)^{*}, x, u \in G$, and set $\psi=* \chi$. In either case $\psi$ is an isomorphism such that $\overline{x^{\psi}}=\bar{x}^{\alpha}$ for all $x \in[K, K]$.

\section{Proof of Theorem 1.1}

Sections 5 and 6 were devoted to the proof of Theorem 1.1 in the special situation where $R$ and $S$ were their own super *-closures (Theorem 5.2 in the 
non-PI case and Theorem 6.2 in the PI case). In this section, we complete the proof of Theorem 1.1. We refer the reader to its statement in Section 1.

Proof of Theorem 1.1. Let $\alpha: \overline{[K, K]} \rightarrow \overline{[L, L]}$ be the given Lie isomorphism. We designate the super ${ }^{*}$-closures of $R$ and $S$ by $A=R C_{*} \otimes_{C_{*}} E$ and $B=S D_{*} \otimes_{D_{*}} F$ respectively, noting that the respective skew elements of $A$ and $B$ are $M=K C_{*} \otimes E$ and $N=L D_{*} \otimes F$. By Lemma 2.4, $\alpha$ may be extended to a $\gamma$-semilinear Lie isomorphism (again denoted by) $\alpha: \overline{[M, M]} \rightarrow \overline{[N, N]}$ given by

$$
\overline{\sum x_{i} c_{i} \otimes \lambda_{i}} \longmapsto \overline{\sum y_{i} c_{i}^{\gamma} \otimes \lambda_{i}^{\gamma}},
$$

where $x_{i} \in[K, K], c_{i} \in C_{*}, y_{i} \in[L, L], \bar{y}_{i}=\bar{x}_{i}^{\alpha}$, and $\gamma: E \rightarrow F$ is an extension of $\gamma: C_{*} \rightarrow D_{*}$. We note that the bars in (59) refer to the moduli $[M, M] \cap(C \otimes E)$ and $[N, N] \cap(D \otimes F)$. By Theorems 5.2 and 6.2 , there exists a ring monomorphism $\psi:\langle[M, M]\rangle \rightarrow\langle[N, N]\rangle C_{B}+C_{B}$ such that

$$
\left(\sum x_{i} c_{i} \otimes \lambda_{i}\right)^{\psi}=\sum y_{i} c_{i}^{\gamma} \otimes \lambda_{i}^{\gamma}+\mu
$$

where $x_{i} \in[K, K], c_{i} \in C_{*}, y_{i} \in[L, L], \overline{y_{i}}=\bar{x}_{i}^{\alpha}$, and $\mu \in C_{B} \cap N$. In particular, for $x \in[K, K]$ we have

$$
x^{\psi}=y+\rho_{x, y},
$$

where $y \in[L, L], \bar{y}=\bar{x}^{\alpha}, \rho_{x, y} \in N \cap C_{B}$. From (60), it is easy to see that $[[K, K],[K, K]] \psi=[[L, L],[L, L]]$ and therefore $\psi:\langle[[K, K],[K, K]]\rangle \rightarrow$ $\langle[[L, L],[L, L]]\rangle$ is an isomorphism. By $[10$, Theorem 3.4], $\langle[[K, K],[K, K]]\rangle$ and $\langle[[L, L],[L, L]]\rangle$ contain nonzero *-ideals $I$ and $J$ of $R$ and $S$ respectively. Clearly, $I^{\psi} \supseteq J I^{\psi} J$, thus proving the last part of Theorem 1.1.

We now return to $(60)$ with the aim of showing that $\rho_{x, y} \in L D_{*} \cap D$. We begin by choosing a $D_{*}$-basis $\left\{\mu_{i}\right\}$ for $F$. Since $N=\sum_{i} L D_{*} \otimes \mu_{i}$ and $C_{B}=\sum_{i} D \otimes \mu_{i}$, and since $\rho_{x, y} \in N \cap C_{B}$, we may write

$$
\rho_{x, y}=\sum_{i} d_{i} \otimes \mu_{i}, \quad d_{i} \in L D_{*} \cap D .
$$

Suppose that we can also write

$$
x^{\psi}=y_{0}+\rho_{s, y_{0}},
$$

where

$$
\rho_{x, y_{0}}=\sum_{i} d_{i}^{\prime} \otimes \mu_{i}, \quad d_{i}^{\prime} \in L D_{*} \cap D .
$$


Since $\bar{y}=\bar{x}^{\alpha}=\overline{y_{0}}$, we see that $y=y_{0}+\delta$, where $\delta \in[L, L] \cap D$. Subtraction of (62) from (60) yields

$$
\rho_{x, y}+\delta=\rho_{x, y_{0}}
$$

and (since $\delta$ really means $\delta \otimes 1$ ) we conclude, comparing (61) and (63), that

$$
\sum_{i \neq 1} d_{i} \otimes \mu_{i}=\sum_{i \neq 1} d_{i}^{\prime} \otimes \mu_{i}
$$

whence $d_{i}=d_{i}^{\prime}$ for $i \neq 1$. Thus $x^{\tau}=\sum_{i \neq 1} d_{i} \otimes \mu_{i}$ is independent of the choice of $y$ in (61) and so we may write for all $x \in[K, K]$,

$$
x^{\psi}=x^{\theta}+x^{\tau},
$$

where $x^{\theta}=y+d, y \in[L, L], \bar{y}=\bar{x}^{\alpha}, d \in L D_{*} \cap D$. We have already noted that $\psi$ maps $\langle[[K, K],[K, K]]\rangle$ onto $\langle[[L, L],[L, L]]\rangle \subseteq S \otimes 1$ and that $\langle[[K, K],[K, K]]\rangle$ contains a nonzero *-ideal $I$. Let $x \in[K, K]$ and let $r \in I$, noting that both $r^{\psi}$ and $(x r)^{\psi}$ lie in $S \otimes 1$ and that $x^{\theta} \in D \otimes 1$. Therefore, from $(x r)^{\psi}=x^{\psi} r^{\psi}=x^{\theta} r^{\psi}+x^{\tau} r^{\psi}$, we conclude that $x^{\tau} I^{\psi} \subseteq(S D+D) \otimes 1$. On the other hand, since $x^{\tau}=\sum_{i \neq 1} d_{i} \otimes \mu_{i}$ and $r^{\psi} \in S \otimes 1$, we see that $x^{\tau} r^{\psi} \in$ $\sum_{i \neq 1}(S D+D) \otimes \mu_{i}$. As a result we have $x^{\tau} I^{\psi}=0$. But (as we have previously noted), letting $J_{0}$ be a nonzero ${ }^{*}$-ideal of $S$ contained in $\langle[[L, L],[L, L]]\rangle$, we know that $J=J_{0} I^{\psi} J_{0}$ is a nonzero *-ideal of $S$ contained in $I^{\psi}$. Hence $x^{\tau} J=0$, which forces $x^{\tau}=0$. Thus we may rewrite (65) as

$$
x^{\psi}=y+\rho, \quad x \in[K, K],
$$

where $y \in[L, L], \bar{y}=\bar{x}^{\alpha}, \rho \in L D_{*} \cap D$. This completes the proof of Theorem 1.1.

\section{The Pierce Decomposition}

In this section, we shall discuss how Lie isomorphisms behave relative to a Pierce decomposition in a rather special situation. The results we obtain will prove useful when we analyze the "mixed" case in Section 9, i.e., when $R$ has an involution of the first kind and $S$ has an involution of the second kind.

Let $G$ be a simple GPI ring whose centroid is an algebraically closed field $E$ of char. $\neq 2$ (thus $G$ is a dense ring of linear transformations of finite rank of a vector space $V$ over $E$ ). Furthermore, we assume $G$ has an involution * of the first kind, with $(V: E) \geq 9$ (respectively $(V: E) \geq 6$ ) if $*$ is of transpose type (respectively, of symplectic type) (see [3, p. 157]). In case $*$ is of transpose type, we may pick orthogonal symmetric idempotents $e_{1}, e_{2} \in G$ 
(with $e_{3}$ formally defined to be $1-e_{1}-e_{2}$ ) such that rank $e_{i} \geq 3, i=1,2,3$. In case $*$ is of symplectic type, $e_{1}, e_{2}, e_{3}$ are similarly chosen but with rank $e_{i} \geq 2, i=1,2,3$. In either case we may write $G$ in its Pierce decompostion relative to $e_{1}, e_{2}, e_{3}$ :

$$
G=\oplus \sum_{i, j=1}^{3} G_{i j}, G_{i j}=e_{i} G e_{j} \quad\left(G_{i}=G_{i i}\right) .
$$

We let $M$ denote the skew elements of $G$, noting in our situation that it is well-known that $M=[M, M]$. For $i=1,2,3$, we set $M_{i}=M \cap G_{i}$, also noting that $M_{i}=\left[M_{i}, M_{i}\right]$. For $i \neq j$, we set $M_{i j}=\left\{x_{i j}-x_{i j} \mid x_{i j} \in G_{i j}, x_{j i}=x_{i j}^{*}\right\}$. Clearly then

$$
M=M_{1} \oplus M_{2} \oplus M_{3} \oplus M_{12} \oplus M_{13} \oplus M_{23} .
$$

It is well-known that $M, M_{1}, M_{2}, M_{3}$ are each simple Lie algebras over $F$ [4, Theorem 2.15] and, using this fact, it is easy to prove that

$$
M_{i j}=\left[M_{i j}, M_{j}\right]=\left[M_{i},\left[M_{i j}, M_{j}\right]\right], i \neq j .
$$

Let $H$ be a simple GPI ring whose centroid is an algebraically closed field $F$ of char. $\neq 2$ such that $[H, H] \cap F=0$. By [4, Theorem 1.12], we know that $[H, H]$ is a simple Lie algebra over $F$.

We now suppose there is an isomorphism $\gamma: E \rightarrow F$ and that we have a $\gamma$-semilinear Lie isomorphism $\alpha: M \rightarrow[H, H]$. For $i=1,2,3$, we set $N_{i}=$ $M_{i}^{\alpha}$, and for $i \neq j$, we set $N_{i j}=M_{i j}^{\alpha}$. Applying $\alpha$ to (66), we obtain the decomposition

$$
[H, H]=N_{1} \oplus N_{2} \oplus N_{3} \oplus N_{12} \oplus N_{13} \oplus N_{23}
$$

and applying $\alpha$ to $M_{i}=\left[M_{i}, M_{i}\right]$ and to (67), we obtain

$$
N_{i}=\left[N_{i}, N_{i}\right], N_{i j}=\left[N_{i j}, N_{j}\right]=\left[N_{i},\left[N_{i j}, N_{j}\right]\right], i \neq j .
$$

Also it is clear that $\left[N_{i}, N_{j}\right]=0$ for $i \neq j$. We come now to the main result of this section.

Lemma 8.1. $N_{i} N_{j}=0$ for $i \neq j$.

Proof. We may assume $i=1, j=2$. Choose $m_{i} \neq 0 \in M_{i}, i=1,2,3$, and set $a=m_{1}^{\alpha}, b=m_{2}^{\alpha}, c=m_{3}^{\alpha}$, noting that $1, a, b, c$ are $F$-independent. For all $x \in M$, we see that

$$
\left[\left[\left[x, m_{1}\right], m_{2}\right], m_{3}\right]=0
$$


and, applying $\alpha$ to (70) and setting $y=x^{\alpha}$, we obtain

$$
[[[y, a], b], c]=0
$$

for all $y \in[H, H]$. By Lemma 3.2, there exists $v \in[H, H]$ such that $v a \notin[H, H]$ and by Lemma 3.1 we know that $H=[H, H]+F v a$. Since

$$
[[[v a, a], b], c]=[[[v, a], b], c] a=0,
$$

we then conclude that

$$
[[[y, a], b], c]=0
$$

for all $y \in H$. As is well known, (71) translates to the following tensor product relation

$1 \otimes a b c-a \otimes b c-b \otimes a c-c \otimes a b+a b \otimes c+a c \otimes b+b c \otimes a-a b c \otimes 1=0$.

By Lemma 3.3, we see in particular that

$$
a b=\beta+\delta a+\lambda b+\mu c, \beta, \delta, \lambda, \mu \in F .
$$

We pick $x \in M_{3}$ such that $\left[m_{3}, x\right] \neq 0$ and, setting $y=x^{\alpha}$ and applying $\alpha$, we see that $[c, y] \neq 0$. We commute $y$ with (72) to obtain $0=\mu[c, y]$, whence $\mu=0$ and (72) becomes

$$
a b=\beta+\delta a+\lambda b .
$$

Now pick $y \in N_{2}$ and commute $y$ with (73) to obtain

$$
(a-\lambda)[b, y]=0 .
$$

Next we pick $w \in N_{1}$ and commute $w$ with (74) to obtain

$$
[a, w][b, y]=0 .
$$

We have thus proved that $\left[N_{1}, N_{1}\right]\left[N_{2}, N_{2}\right]=0$, but, since $N_{i}=\left[N_{i}, N_{i}\right]$, we finally have shown that $N_{1} N_{2}=0$

Corollary 8.2. $N_{i} N_{j k}=0$ for $i, j, k$ distinct.

Proof. We know from (69) that $N_{j k}=\left[N_{j k}, N_{k}\right]$. Now pick $m_{i} \in M_{i}, m_{k} \in$ $M_{k}$, and $x_{j k} \in G_{j k}$. Repeated use of Lemma 8.1 then shows that

$$
\begin{aligned}
m_{i}^{\alpha}\left[\left(x_{j k}-x_{k j}\right)^{\alpha}, m_{k}^{\alpha}\right] & =m_{i}^{\alpha}\left(x_{j k}-x_{k j}\right)^{\alpha} m_{k}^{\alpha} \\
& =\left[m_{i}^{\alpha},\left(x_{j k}-x_{k j}\right)^{\alpha}\right] m_{k}^{\alpha} \\
& =\left[m_{i}, x_{j k}-x_{k j}\right]^{\alpha} m_{k}^{\alpha} \\
& =0 .
\end{aligned}
$$




\section{Proof of Theorem 1.3}

This entire section is devoted to the proof of Theorem 1.3. We shall not repeat its statement here but simply refer the reader to Section 1 . We are therefore assuming that $R$ is a ${ }^{*}$-prime ring of type 1 with skew elements $K$, that $S$ is a ${ }^{*}$-prime ring of type 2 with skew elements $L$, and that one of $R$ and $S$ is GPI. By Theorem 4.1, both $R$ and $S$ are GPI. We are given a Lie isomorphism

$$
\alpha:[K, K] \rightarrow \overline{[L, L]},
$$

where $[K, K]$ and $\overline{[L, L]}$ are each assumed to be nonzero (and hence are prime Lie rings). Our aim in this section is to show that such a Lie isomorphism cannot exist except in a couple of low-dimensional situations. Let $A=R C_{*} \otimes_{C_{*}} E$ and $B=S D_{*} \otimes_{D_{*}} F$ be the respective super *-closures of $R$ and $S$. Then $P=K C_{*} \otimes_{C_{*}} E$ and $Q=L D_{*} \otimes_{D *} F$ are the respective skew elements of $A$ and $B$. We know by Theorem 2.4 that $\alpha$ may be extended to a $\gamma$-semilinear Lie isomorphism (again denoted by $\alpha$ )

$$
\alpha:[P, P] \rightarrow[Q, Q],
$$

where $\gamma: E \rightarrow F$ is a suitable isomorphism. Let $G$ be the socle of $A$ and let $H \oplus H^{*}$ be the ${ }^{*}$-socle of $B$. Let $M$ denote the skew elements of $G$ (we note here that $M=[M, M])$, and let $N=\left\{y-y^{*} \mid y \in H\right\}$ denote the skew elements of $H \oplus H^{*}$. It is straightforward to show that $M$ is the unique minimal Lie ideal of $P$ and that $\overline{[N, N]}$ is the unique minimal Lie ideal of $\overline{[Q, Q]}$. Therefore, $\alpha$ must induce a Lie isomorphism

$$
\alpha: M \rightarrow \overline{[N, N]},
$$

where we shall write

$$
x^{\alpha}=\overline{y-y^{*}}, x \in M, y \in[H, H] .
$$

Furthermore, there is a natural Lie isomorphism

$$
\nu: \overline{[N, N]} \rightarrow \overline{[H, H]}
$$

given by

$$
\overline{y-y^{*}} \longmapsto \bar{y}, y \in[H, H] .
$$

Then the composite $\beta=\alpha \nu$ is a $\gamma$-semilinear Lie isomorphism

$$
\beta: M \rightarrow \overline{[H, H]}
$$

given by 


$$
x^{\beta}=\bar{y}, x \in M,
$$

where $x^{\alpha}=\overline{y-y^{*}}$.

We first dispose of the situation in which $[H, H] \cap F \neq 0$. As we have seen earlier, this forces $H$ to be PI (and hence $H=M_{k}(F)$ ), char. $F=p \geq 3, p$ divides $k$, and $[H, H] \supseteq F 1$. Since $H$ is PI, we know that $G$ must be PI (and hence $G=M_{n}(E)$ ). The dimension of $\overline{[H, H]}$ over $F$ is clearly $k^{2}-2$. The involution in $G$ is either of transpose type or of symplectic type.

If * is of transpose type in $G$, then $\operatorname{dim} M=\left(n^{2}-n\right) / 2$. Equating $\operatorname{dim} M$ with $\operatorname{dim} \overline{[H, H]}$, we have $\left(n^{2}-n\right) / 2=k^{2}-2$, which results in the quadratic equation in $n$ :

$$
n^{2}-n-2(k-2)=0
$$

For $n$ to be an integral solution of $(77)$, the discriminant $1+8\left(k^{2}-2\right)$ equals $q^{2}$ for some integer $q$, whence $8 k^{2}-15=q^{2}$.

If $*$ is of symplectic type, $n=2 m$ and $\operatorname{dim} P=2 m^{2}+m$. For the equation

$$
2 m^{2}+m=k^{2}-2
$$

to have an integral solution for $m$, one is again led to $1+8\left(k^{2}-2\right)=q^{2}$ for some integer $q$ whence as before

$$
8 k^{2}-15=q^{2} .
$$

But we now claim that the equation $8 k^{2}-15=q^{2}$ has no integral solutions. Indeed, if it did, there would also be a solution mod 9 of

$$
y^{2}-3=-x^{2} .
$$

The squares mod 9 are $0,1,4,7$ and so the values of $y^{2}-3 \bmod 9$ are $1,4,6,7$. However, the values of $-x^{2} \bmod 9$ are $0,2,5,8$, which leaves us with the desired contradiction.

We may therefore assume for the remainder of this section that $[H, H] \cap F=$ 0, i.e., $\overline{[H, H]}=[H, H]$. We note that $(75)$ becomes

$$
x^{\alpha}=y-y^{*}, x \in P, y \in[H, H],
$$

and that (76) becomes

$$
x^{\beta}=y, x \in M, y \in[H, H], \beta=\alpha \nu .
$$

We first consider the situation when $G$ (and hence $H$ ) is PI and the dimension $(G: E)$ (and hence $(H: F)$ ) is low. We know that $G=M_{n}(E)$ and $H=$ $M_{k}(F)$. Clearly, $([H, H]: F)=k^{2}-1$, in which case the values of $([H, H]$ : 
$F)=k^{2}-1$ are $3,8,15,24$ for $k=2,3,4,5$ respectively. If * in $G$ is of transpose type, then $(P: E)=\left(n^{2}-n\right) / 2$ and the values of $(M: E)$ are $3,6,10,15,21,28$ for $n=3,4,5,6,7,8$ respectively. If $*$ in $G$ is of symplectic type then $n=2 m$, $(P: E)=2 m^{2}+m$, and so the values of $(P: E)$ are 3,10 for $m=1,2$ respectively. Equating $(P: E)$ and $([H, H]: F)$, we find that only two cases occur in which $P$ and $[H, H]$ could possibly be Lie isomorphic:

1. $m=1, n=3, k=2$,

2. $n=6, k=4$.

These possibilites do in fact occur [7, p. 142].

In view of the preceding discussion we may assume without loss of generality that $(G: E) \geq 81$ if $*$ is of transpose type and that $(G: E) \geq 36$ if * is of symplectic type.

We now continue with our analysis of the Lie isomorphism $\beta: M \rightarrow[H, H]$ under these dimension restrictions. The conditions on $M$ and $[H, H]$ are now precisely those assumed in Section 8 (with $\beta$ now playing the role of $\alpha$ ). With reference to the terminology of Section 8, we have $M_{i}=M \cap G_{i}, M_{i j}=$ $\left\{x_{i j}-x_{j i} \mid x_{i j} \in G_{i j}\right\}$ for $i \neq j, i, j=1,2,3, N_{i}=M_{i}^{\beta}, N_{i j}=M_{i j}^{\beta}, i \neq j$. By Lemma 8.1, we have $N_{i} N_{j}=0$ for $i \neq j$, and by Corollary 8.2, we have $N_{i} N_{j k}=0$ for $i, j, k$ distinct.

Our immediate aim now is to construct an isomorphim of $G$ into $H$ and then to show that this leads to a contradiction. In preparation for this, we need the following two lemmas (essentially [9, Lemmas 7.2 and 7.3]).

Lemma 9.1. Let $x_{1}, x_{2}, \ldots x_{n} \in M_{1}, m_{j} \in M_{j}, x_{1 j} \in G_{1 j}, j \neq 1$. Then

$$
x_{1}^{\beta} x_{2}^{\beta} \cdots\left(x_{1 j}-x_{j 1}\right)^{\beta} m_{j}^{\beta}=\left(x_{1} x_{2} \cdots x_{n} x_{1 j}+(-1)^{n+1} x_{j 1} x_{n} \cdots x_{1}\right)^{\beta} m_{j}^{\beta} .
$$

Proof. The proof is by induction on $n$. For $n=1$,

$$
\begin{gathered}
x_{1}^{\beta}\left(x_{1 j}-x_{j 1}\right)^{\beta} m_{j}^{\beta}=\left[x_{1}^{\beta},\left(x_{1 j}-x_{j 1}\right)^{\beta}\right]= \\
{\left[x_{1}, x_{1 j}-x_{j 1}\right]^{\beta} m_{j}^{\beta}=\left(x_{1} x_{1 j}+x_{j 1} x_{1}\right)^{\beta} m_{j}^{\beta}=\left(x_{1} x_{1 j}+(-1)^{2} x_{j 1} x_{1}\right)^{\beta} m_{j}^{\beta} .}
\end{gathered}
$$

Assuming the lemma is true for $n$, we see that

$$
\begin{aligned}
& x_{1}^{\beta} x_{2}^{\beta} \cdots\left(x_{1 j}-x_{j 1}\right)^{\beta} m_{j}^{\beta} \\
& =\left[x_{1}^{\beta},\left(x_{2} \cdots x_{n+1} x_{1 j}+(-1)^{2} x_{j 1} x_{n+1} \cdots x_{2}\right)^{\beta}\right] m_{j} \\
& =\left(x_{1} x_{2} \cdots x_{n+1} x_{1 j}+(-1)^{n+2} x_{j 1} x_{n+1} \cdots x_{1}\right)^{\beta} m_{j}^{\beta} .
\end{aligned}
$$


Lemma 9.2. If $\sum x_{1} x_{2} \cdots x_{n}=0, x_{I} \in M_{i}$, then $b=\sum x_{1}^{\beta} x_{2}^{\beta} \cdots x_{n}^{\beta}=0$.

Proof. We first note that $0=0^{*}=\sum(-1)^{n} x_{n} \cdots x_{2} x_{1}=0$. In view of (8.3), (8.4) and Corollary 8.2, it is clear that the Lie subring generated by $N_{12} \cup N_{13} \cup N_{23}$ is a Lie ideal of $[H, H]$ and hence equal to $[H, H]$. Therefore, in order to show that $b=0$, it suffices to show that $b N_{i j}=0, i \neq j$. By Corollary 8.2, without loss of generality we need only show that $b N_{12}=0$. Furthermore, since $N_{12}=\left[N_{12}, N_{2}\right]$ by (8.4), we see in light of Corollary 8.2 that it suffices to show that $b\left(x_{12}-x_{21}\right)^{\beta} m_{2}^{\beta}=0, x_{12} \in G_{12}, m_{2} \in M_{2}$. But by Lemma 9.1 ,

$$
\begin{aligned}
b\left(x_{12}\right. & \left.-x_{21}\right)^{\beta} m_{2}^{\beta}=\sum\left(x_{1} x_{2} \cdots x_{n} x_{12}+(-1)^{n+1} x_{21} x_{n} \cdots x_{1}\right)^{\beta} m_{2}^{\beta} \\
& =\left\{\left(\sum x_{1} x_{2} \cdots x_{n}\right) x_{12}-x_{21}\left(\sum(-1)^{n} x_{n} \cdots x_{1}\right)\right\}^{\beta} m_{2}^{\beta}=0
\end{aligned}
$$

and the proof is complete.

Lemma 9.2. enables us to define a ring hommorphism $\sigma$ of $G_{1}=\left\langle M_{1}\right\rangle$ onto $N_{1}$ and, since $G_{1}$ is simple, $\sigma$ must in fact be an isomorphism.

We now return to the Lie isomorphism $\alpha$, recalling in particular that for all $x \in M_{1}$,

$$
x^{\alpha}=y-y^{*}, y=x^{\beta} \in N_{1} .
$$

We have just seen that $\left\langle N_{1}\right\rangle=G^{\sigma}$ is a simple ring contained in $H$. We denote by $W$ the skew elements of the ${ }^{*}$-simple ring $\left\langle N_{1}\right\rangle \oplus\left\langle N_{1}\right\rangle^{*}$.

Lemma 9.3. $M_{1}^{\alpha}=W$.

Proof. Clearly, $M_{1}^{\alpha} \subseteq W$. Now let $w \in W$. On the one hand,

$$
w=\sum\left(y_{1} y_{2} \cdots y_{n}-y_{n}^{*} \cdots y_{1}^{*}\right), y_{i} \in N_{1} .
$$

On the other hand, we see from (68) that

$$
w=z_{1}-z_{1}^{*}+z_{2}-z_{2}^{*}+z_{3}-z_{3}^{*}+z_{12}-z_{12}^{*}+z_{13}-z_{13}^{*}+z_{23}-z_{23}^{*},
$$

where $z_{i} \in N_{i}$ and $z_{i j} \in N_{i j}$. It follows that

$$
\sum y_{1} y_{2} \cdots y_{n}=z_{1}+z_{2}+z_{3}+z_{12}+z_{23},
$$

which we rewrite as

$$
\sum y_{1} y_{2} \cdots y_{n}=z_{1}+m_{2}^{\beta}+m_{3}^{\beta}+x_{12}^{\beta}+x_{13}^{\beta}+x_{23}^{\beta} .
$$


Let $u \in M_{2}$ and commute (81) with $u^{\beta}$. This leaves us with

$$
0=0=\left[m_{2}, u\right]^{\beta}+\left[x_{12}, u\right]^{\beta}=\left[x_{23}, u\right]^{\beta},
$$

whence $\left[m_{2}, u\right]=\left[x_{12}, u\right]=\left[x_{23}, u\right]^{\beta}=0$ for all $u \in M_{2}$. Since $\left\langle M_{1}\right\rangle=G_{1}$, it follows easily that $m_{2}=x_{12}=x_{23}=0$. Similarly, by commuting (81) with $v^{\beta}, v \in M_{3}$, we find that $m_{3}=x_{13}=0$. Therefore, $w=z_{1}-z_{1}^{*} \in M_{1}^{\alpha}$ and the proof is complete.

We now define a map $\psi: G_{1} \rightarrow\left\langle N_{1} \oplus N_{1}^{*}\right\rangle$ according to the rule

$$
\sum x_{1} x_{2} \cdots x_{n} \longmapsto \sum\left(y_{1}-y_{1}^{*}\right) \cdots\left(y_{n}-y_{n}^{*}\right)
$$

where $x \in M_{1}, x_{i}^{\alpha}=y_{i}-y_{i}^{*}$. We remark that $y_{i}=x_{i}^{\beta}=x_{i}^{\sigma}$, where $\sigma$ is the isomorphism of $G_{1}$ onto $\left\langle N_{1}\right\rangle$ previously defined. To show $\psi$ is well-defined, suppose

$$
\sum x_{1} x_{2} \cdots x_{n}=0
$$

Applying $\sigma$ to (82), we see that $\sum y_{1} y_{2} \cdots y_{n}=0$. Applying * to (82), we have

$$
\sum(-1)^{n} x_{n} \cdots x_{2} x_{1}=0 .
$$

Applying Lemma 9.1 to (83), we have

$$
\sum(-1)^{n} y_{n} \cdots y_{2} y_{1}=0
$$

which becomes, after an application of $*$,

$$
\sum(-1)^{n} y_{1}^{*} y_{2}^{*} \cdots y_{n}^{*}=0 .
$$

We conclude that $\sum\left(y_{1}-y_{1}^{*}\right) \cdots\left(y_{n}-y_{n}^{*}\right)=0$ and therefore $\psi$ is a welldefined ring homomorphism, which extends the restriction of $\alpha$ to $M_{1}$. It is clear that $G_{1}^{\psi}=\left\langle M_{1}\right\rangle^{\psi}=\left\langle M^{\alpha}\right\rangle$. But by Lemma $9.3, M_{1}^{\alpha}=W$, the skew elements of $\left\langle N_{1}\right\rangle \oplus\left\langle N_{1}\right\rangle^{*}$, and since $\left\langle N_{1}\right\rangle \oplus\left\langle N_{1}\right\rangle^{*}$ is $*_{\text {-simple, we know that }}$ $\left\langle M_{1}^{\alpha}\right\rangle=\left\langle N_{1}\right\rangle \oplus\left\langle N_{1}\right\rangle^{*}$. Hence $\psi$ is an isomorphism of $G$ onto $\left\langle N_{1}\right\rangle \oplus\left\langle N_{1}\right\rangle^{*}$. This is an obvious contradiction, and we can only conclude that in this situation, where $[H, H] \cap F=0, M$ cannot be Lie isomorphic to $[H, H]$.

The proof of Theorem 1.3 is now complete.

\section{REFERENCES}

1. W. E. Baxter and W. S. Martindale, 3rd, The extended centroid in semiprime rings, Comm. Algebra 13 (1985), 945-985. 
2. K. I. Beidar, M. Bresar, M. Chebotar and W. S. Martindale, 3rd, in preparation.

3. K. I. Beidar, W. S. Martindale, 3rd, and A. V. Mikhalev, Rings with Generalized Identities, Pure and Applied Mathematics, Marcel Dekker, New York, 1996.

4. I. N. Herstein, Topics in Ring Theory, Univ. of Chicago Press, Chicago, 1969.

5. I. N. Herstein, On the Lie structure of an associative ring, J. Algebra 14 (1970), 561-571.

6. R. A. Howland, Lie isomorphisms of derived rings of simple rings, Trans. Amer. Math. Soc. 145 (1969), 383-396.

7. N. Jacobson, Lie Algebras, Interscience Tracts in Pure and Applied Math., no. 10, Interscience, New York, 1962.

8. W. S. Martindale, 3rd, Rings with involution and polynomial identities, $J$. Algebra 11 (1969), 186-194.

9. W. S. Martindale, 3rd, Lie isomorphisms of the skew elements of a prime ring with involution, Comm. Algebra 4 (1976), 929-977.

10. W. S. Martindale, 3rd and C. R. Miers, Herstein's Lie theory revisited, J. Algebra 98 (1986), 14-37.

11. M. P. Rosen, Isomorphisms of a certain class of prime Lie rings, J. Algebra 89 (1984), 291-317.

Philip S. Blau

College of General Studies, Boston University

Boston MA 02215, U.S.A.

Wallace S. Martindale, 3rd

Department of Mathematics, University of Massachusetts

Amherst MA 01003, U.S.A. 